\title{
Reference Tables for Thermocouples of Iridium-Rhodium Alloys Versus Iridium
}

\author{
G. F. Blackburn and F. R. Caldwell
}

(September 11, 1963)

\begin{abstract}
The program at the National Bureau of Standards to establish reference tables of temperature versus emf for thermocouples of iridium-rhodium alloys versus iridium has been extended to cover all three of the currently used thermocouples of this type. In addition to the values published in 1962 for the $40 \mathrm{Ir}-60 \mathrm{Rh}$ versus Ir thermocouples, tables now are available for thermocouples of $60 \mathrm{Ir}-40 \mathrm{Rh}$ and $50 \mathrm{Ir}-50 \mathrm{Rh}$ versus Ir. These tables give emfs for temperatures in degrees Celsius from 0 to $2150{ }^{\circ} \mathrm{C}$ and in degrees Fahrenheit from 32 to $3900{ }^{\circ} \mathrm{F}$, and temperatures in these units with emf in millivolts as the argument.

In addition to the reference tables for these thermocouples, temperature-emf relationships are presented for other alloys containing 10, 25, 75, and 90 percent iridium versus iridium. It appears from the information obtained on all of the alloy versus iridium combinations tested that the $50 \mathrm{Ir}-50 \mathrm{Rh}$ alloy versus iridium gives about the maximum thermal emf $\left(12.2\right.$ millivolts at $\left.2150^{\circ} \mathrm{C}\right)$, and as a result may provide the optimum thermocouple combination of this type.
\end{abstract}

\section{Introduction}

A program to establish reference tables of thermal emf of a number of alloys of iridium and rhodium versus iridium has been in progress at the National Bureau of Standards for several years. Tables for 40 percent iridium-60 percent rhodium versus iridium were published in 1962 [1]. ${ }^{1}$ The present paper includes tables for all the other alloys of the series studied at NBS viz, 10, 25, 40, 50, 75, and 90 percent rhodium. ${ }^{2}$

\section{Apparatus and Procedure}

The apparatus and procedure have been described previously [1]. Briefly, measurements of thermal emf were made at temperatures up to $2500{ }^{\circ} \mathrm{F}^{3}$ $\left(2000{ }^{\circ} \mathrm{F}\right.$ in a few cases) using a conventional platinum-wound furnace. Temperatures in the furance were measured with a platinum-10 percent rhodium versus platinum thermocouple. Temperatures above the limit of this furnace were obtained in an iridium blackbody heated by electrical induction. A conventional optical pyrometer, modified to permit finer adjustment of current through the lamp filament, was used to measure temperature. The calibration of the pyrometer gave temperature as a function of lamp current.

Each thermocouple wire was annealed before testing by heating it electrically in air for about 1 min at approximately $400{ }^{\circ} \mathrm{F}$ below its melting point. The junction was welded in an oxyacetylene flame.

\footnotetext{
1 Figures in brackets indicate the literature references at the end of this paper. 2 For brevity, the expression "25 percent Rh," for example, will be used to indicate a thermocouple in which the positive leg is 75 percent $\mathrm{Ir}-25$ percent $\mathrm{Rh}$, indicate a thermocouple in

and the negative is iridium. of 1948 .
}

\section{Thermocouples}

Table 1 is a list of the thermocouples used, in order of increasing percentage of rhodium in the alloy leg. The letter in a thermocouple number designates the lot from which the wire was taken, and refers to a particular shipment received from the manufacturer. The first digit refers to the composition of the alloy as follows: 1 signifies 10 percent; 2, 25 percent; 4, 40 percent; 5, 50 percent; 7, 75 percent; and 9, 90 percent rhodium. The last two digits are the wire diameter in thousandths of an inch. Three lots of wire (A, D, and F) were supplied by the Sigmund Cohn Corporation, and two (C and $\mathrm{G}$ ) by Engelhard Industries, Inc. Since lot D consisted of alloy wire only, iridium to pair with alloy wires from this lot was taken from other lots.

TABle 1. Description of thermocouples

\begin{tabular}{|c|c|c|c|}
\hline $\begin{array}{l}\text { Percent } \\
\text { Rh }\end{array}$ & $\begin{array}{c}\text { Thermocouple } \\
\text { No. }\end{array}$ & $\begin{array}{l}\text { Diameter of } \\
\text { wire in. }\end{array}$ & Manufacturer \\
\hline 10 & $\begin{array}{l}\text { A } 135 \\
\text { G120 }\end{array}$ & $\begin{array}{r}0.035 \\
.020\end{array}$ & $\begin{array}{l}\text { Sigmund Cohn } \\
\text { Engelhard }\end{array}$ \\
\hline 25 & $\begin{array}{l}\mathrm{A} 235 \\
\mathrm{G} 220\end{array}$ & $\begin{array}{l}.035 \\
.020\end{array}$ & $\begin{array}{l}\text { Sigmund Cohn } \\
\text { Engelhard }\end{array}$ \\
\hline 40 & $\begin{array}{cc} & \mathrm{A} 435 \\
& \mathrm{C} 420 \\
1 & \mathrm{D} 420 \mathrm{C} \\
2 & \mathrm{D} 435 \mathrm{~B}\end{array}$ & $\begin{array}{l}.035 \\
.020 \\
.020 \\
.035\end{array}$ & $\begin{array}{l}\text { Sigmund Cohn } \\
\text { Engelhard } \\
\text { Sigmund Cohn } \\
\text { Sigmund Cohn }\end{array}$ \\
\hline 50 & $\begin{array}{l}\text { A535 } \\
\text { F530 } \\
\text { F5 }\end{array}$ & $\begin{array}{l}.035 \\
.030 \\
.020\end{array}$ & $\begin{array}{l}\text { Sigmund Cohn } \\
\text { Sigmund Cohn } \\
\text { Sigmund Cohn }\end{array}$ \\
\hline 75 & $\begin{array}{l}\text { A735 } \\
\text { G720 }\end{array}$ & $\begin{array}{l}.035 \\
.020\end{array}$ & $\begin{array}{l}\text { Sigmund Cohn } \\
\text { Engelhard }\end{array}$ \\
\hline 90 & $\begin{array}{l}\text { A } 935 \\
\text { G920 }\end{array}$ & $\begin{array}{l}.035 \\
.020\end{array}$ & $\begin{array}{l}\text { Sigmund Cohn } \\
\text { Engelhard }\end{array}$ \\
\hline
\end{tabular}

${ }^{1}$ Iridium wire from lot $\mathrm{C}$.

${ }_{2}$ Iridium wire from lot B (comprising $60 \mathrm{Rh}$ and $\mathrm{Ir}$ wire). 


\section{Computations}

For each alloy tested, the emf at a given temperature was taken as the average of the emfs of the different lots of that alloy. However, if a lot contained wires of more than 1 diam, the emf of each size wire was used as if it represented a different lot. For example, the emf for the 40 percent $\mathrm{Rh}$ thermocouple was taken as the average of the emfs of four: lot $\mathrm{A}$, lot $\mathrm{C}$, and the two wire sizes of lot D. With one exception each lot was represented by one thermocouple of a given alloy. Two thermocouples were used from lot $\mathrm{C}$, and the mean of the emfs was taken as the emf for the lot.

The data for the alloys $25,40,50$, and 75 percent rhodium were processed by the Computation Section of the NBS Applied Mathematics Division. An IBM 7090 Computer, employing programing procedures called OMNITAB [2], was used. With the object of finding a functional relation between emf and temperature such that tables could be generated entirely by a digital computer, trials were made in fitting polynominals of various degrees to the observed emfs for the 40 percent $\mathrm{Rh}$ thermocouple. It was determined that the data could be adequately represented by two equations, one of fourth degree for the range $32{ }^{\circ} \mathrm{F}$ to $1000{ }^{\circ} \mathrm{F}$, the second of fifth degree for the range 1000 to 3900 ${ }^{\circ} \mathrm{F}$. In computing the equation for the high range, the data from 1000 to $2500{ }^{\circ} \mathrm{F}$ were weighted 10 times those above $2500{ }^{\circ} \mathrm{F}$, to take account of the higher precision that could be achieved at the lower temperatures.

The emf given for $32{ }^{\circ} \mathrm{F}$ by the equation for the lower range was less than $1 \mu \mathrm{v}$, and the difference between the emfs given by the two equations at the range juncture was also less than $1 \mu \mathrm{v}$. Deviations from observed emfs were randomly distributed, and were small - less than $5 \mu \mathrm{v}$ at temperatures up to about $3000^{\circ} \mathrm{F}$, and only a few were over $10 \mu \mathrm{v}$ at the highest temperatures. Evidently, the equations as computed represented the observed data adequately, and could be used to generate the desired tables. However, it seemed desirable that the equation yield zero emf at $32^{\circ} \mathrm{F}$; and since this could be accomplished by changing the constant term by a fraction of a microvolt, this change was made. Similarly, the equation for the upper range was made to yield the same emf at the juncture of the two ranges as the modified equation for the lower range by changing the constant term slightly.

The procedure followed for the 40 percent $\mathrm{Rh}$ was found equally suitable for the 25,50 , and 75 percent $\mathrm{Rh}$ thermocouples. No change was as great as $1 \mu \mathrm{v}$ except that for the 75 percent $\mathrm{Rh}$ thermocouple, which was $1.4 \mu \mathrm{v}$ at $1000^{\circ} \mathrm{F}$.

The equations for the upper and lower ranges did not give the same value of $d E / d T$ at the juncture for any of the thermocouples. However, they were so nearly the same that the equations yielded the same emf to within $1 \mu \mathrm{v}$ at temperatures up to $20^{\circ} \mathrm{F}$ on either side of the juncture. ${ }^{4}$

${ }^{4}$ It is feasible to use a computer program which performs the curve fitting in such a way that the conditions to be satisfied include zero emf at $32^{\circ} \mathrm{F}$ and equality of both the emfs and the first derivatives at the juncture of the ranges.

\section{Results}

The derived polynominal equations were used to generate tables of emf with ${ }^{\circ} \mathrm{F}$ as the argument (values in tables $4-\mathbf{A}, 8-\mathbf{A}$, and for the alloys 25 and 75 percent $\mathrm{Rh}$ in table $9-\mathrm{A})$. The coefficients of these equations are listed in table 2. For the 40 percent $\mathrm{Rh}$ and 50 percent $\mathrm{Rh}$, the tables are fully developed, giving temperature in ${ }^{\circ} \mathrm{C}$ and ${ }^{\circ} \mathrm{F}$ with emf in 0.01-mv increments as the argument, and emf with ${ }^{\circ} \mathrm{C}$ and ${ }^{\circ} \mathrm{F}$ as the argument, in 10-deg increments. The computer techniques involved conversion from ${ }^{\circ} \mathrm{C}$ to ${ }^{\circ} \mathrm{F}$ and interpolation.

Table 9-A gives emfs for the $10,25,75$, and 90 percent $\mathrm{Rh}$ thermocouples in $100{ }^{\circ} \mathrm{F}$ increments.

Electromotive force versus temperature curves for the alloys considered in this study are shown in figure 1. Figure 2 is a plot of $d E / d T$, derived by machine computation except for the 10 and 90 percent Rh thermocouples. Figure 3 shows the variation of emf with composition of the alloy leg for temperatures $2000,2500,3000$, and $3500{ }^{\circ} \mathrm{F}$. The emf at $2000{ }^{\circ} \mathrm{F}$ for 100 percent $\mathrm{Rh}$ is found from data by Caldwell [3] for $\mathrm{Rh}$ versus Pt27, together with a table of emfs for Ir versus Pt27 given in reference [1]. The point at $2500^{\circ} \mathrm{F}$ is an extrapolation of Caldwell's data.

If the cold junction is at a temperature higher than $32{ }^{\circ} \mathrm{F}$, the appropriate correction can be found by interpolating in the tables, or by the use of the corresponding equation. For the case where copper lead wires are used with the 40 or 50 percent Rh thermocouple the correction can be obtained from table 10-A, which gives the emfs of these alloys and iridium

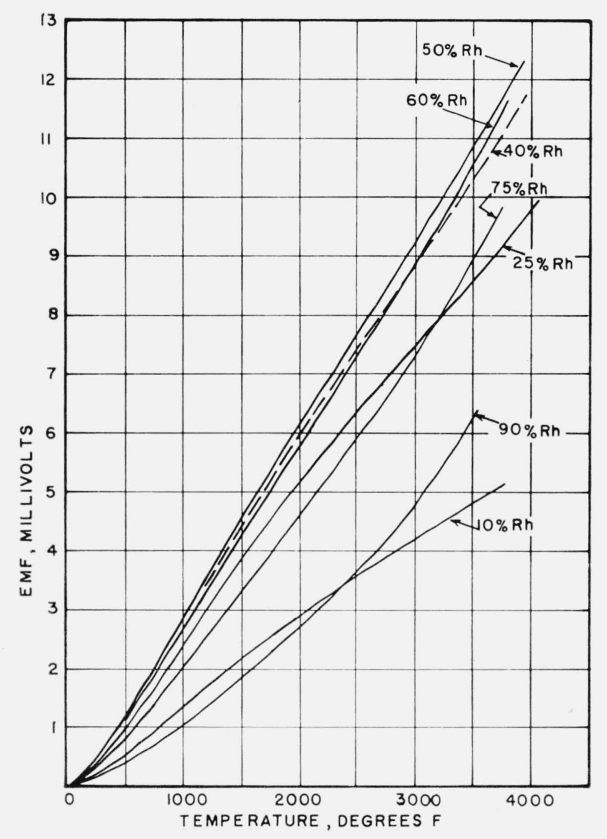

FIGURE 1. Thermal emf of iridium-rhodium alloys against iridium. 
TABLE 2. Values of coefficients in the equation

$\mathrm{E}=\mathrm{A}+\mathrm{BT}+\mathrm{CT}^{2}+\mathrm{DT}^{3}+\mathrm{ET}^{4}+\mathrm{FT}^{5}$

for four IrRh versus Ir thermocouples*

\begin{tabular}{|c|c|c|c|c|}
\hline \multirow{2}{*}{ Coefficient } & \multicolumn{2}{|c|}{$60 \mathrm{Jr}-40 \mathrm{Rh}$} & \multicolumn{2}{|c|}{$50 \mathrm{Ir}-50 \mathrm{Rh}$} \\
\hline & $32^{\circ} \mathrm{F}$ to $1000^{\circ} \mathrm{F}$ & $1000^{\circ} \mathrm{F}$ to 3900 & $32^{\circ} \mathrm{F}$ to $1100^{\circ} \mathrm{F}$ & $\begin{array}{c}1100^{\circ} \mathrm{F} \text { to } 3900 \\
{ }^{\circ} \mathrm{F}\end{array}$ \\
\hline \multirow[t]{3}{*}{$\begin{array}{l}\mathrm{A} \\
\mathrm{B}_{-} \\
\mathrm{P}_{2} \\
\mathrm{D}_{\ldots} \\
\mathrm{F}_{\ldots}\end{array}$} & $\begin{array}{r}-0.052816 \\
1.581377 \cdot 10^{-3} \\
2.196393 \cdot 10^{-6} \\
-1.136843 \cdot 10^{-9} \\
-0.204061 \cdot 10^{-12}\end{array}$ & $\begin{array}{r}-0.353350 \\
2.406678 \cdot 10^{-3} \\
1.366226 \cdot 10^{-6} \\
-0.791802 \cdot 10^{-9} \\
.178246 \cdot 10^{-12} \\
-.013826 \cdot 10^{-15}\end{array}$ & $\begin{array}{r}-0.054717 \\
\quad 1.640880 \cdot 10^{-3} \\
2.193392 \cdot 10^{-6} \\
-1.127263 \cdot 10^{-9} \\
-0.202907 \cdot 10^{-12}\end{array}$ & $\begin{array}{r}-0.143581 \\
1.877703 \cdot 10^{-3} \\
1.981642 \cdot 10^{-6} \\
-1.091919 \cdot 10^{-9} \\
0.251008 \cdot 10^{-12} \\
-.020415 \cdot 10^{-15}\end{array}$ \\
\hline & \multicolumn{2}{|c|}{$75 \mathrm{Ir}-25 \mathrm{Rh}$} & \multicolumn{2}{|c|}{$25 \mathrm{Ir}-75 \mathrm{Rh}$} \\
\hline & $32^{\circ} \mathrm{F}$ to $1000^{\circ} \mathrm{F}$ & $1000^{\circ} \underset{\mathrm{F}}{\mathrm{F}}$ to 4000 & $32^{\circ} \mathrm{F}$ to $1000^{\circ} \mathrm{F}$ & $1000^{\circ} \mathrm{F}$ to 3700 \\
\hline $\begin{array}{l}\mathrm{A} \\
\mathrm{B} \\
\mathrm{C} \\
\mathrm{D} \\
\mathrm{E} \\
\mathrm{F}\end{array}$ & $\begin{array}{r}-0.043211 \\
1.284913 \cdot 10^{-3} \\
2.079622 \cdot 10^{-6} \\
-1.090633 \cdot 10^{-9} \\
0.193374 \cdot 10^{-12} \\
\end{array}$ & $\begin{array}{r}-0.110871 \\
1.449150 \cdot 10^{-3} \\
1.954721 \cdot 10^{-6} \\
-1.097890 \cdot 10^{-9} \\
0.249149 \cdot 10^{-12} \\
-.020193 \cdot 10^{-15}\end{array}$ & $\begin{array}{r}-0.036808 \\
1.191287 \cdot 10^{-3} \\
1.552051 \cdot 10^{-6} \\
-0.683930 \cdot 10^{-9} \\
.092373 \cdot 10^{-1} \\
\end{array}$ & $\begin{array}{r}-0.031682 \\
.979634 \cdot 10^{-3} \\
1.879813 \cdot 10^{-6} \\
-1.042899 \cdot 10^{-9} \\
0.263564 \cdot 10^{-12} \\
-.023458 \cdot 10^{-15}\end{array}$ \\
\hline
\end{tabular}

${ }^{*} \mathrm{E}$ is emf in millivolts; $\mathrm{T}$ is temperature in degrees Fahrenheit.

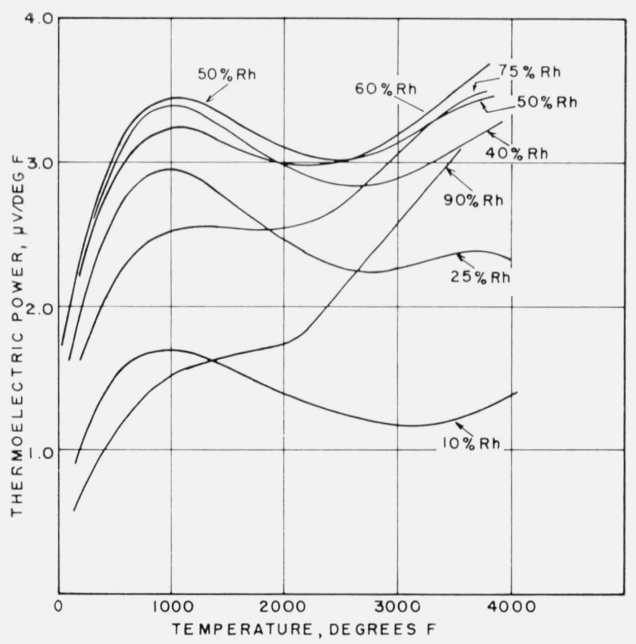

FIgURE 2. Thermoelectric power of iridium-rhodium alloys against iridium.

versus copper for temperatures to $500{ }^{\circ} \mathrm{F}$. This table is particularly useful when the two reference junctions of the thermocouple are at different temperatures.

\section{Discussion}

The maximum difference in emf between thermocouples of a given alloy occurred at the highest temperature associated with each alloy. This maximum for any alloy was that for the 40 percent $\mathrm{Rh}$ thermocouple, about $120 \mu \mathrm{v}$. This is comparable to

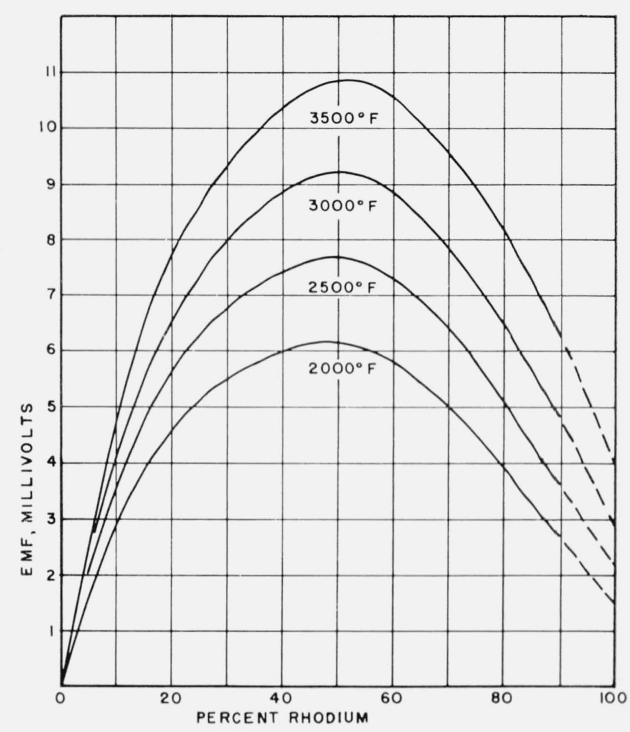

FIgURE 3. Variation at constant temperature of thermal emf with percent rhodium in the alloy legs of iridium-rhodium versus iridium thermocouples.

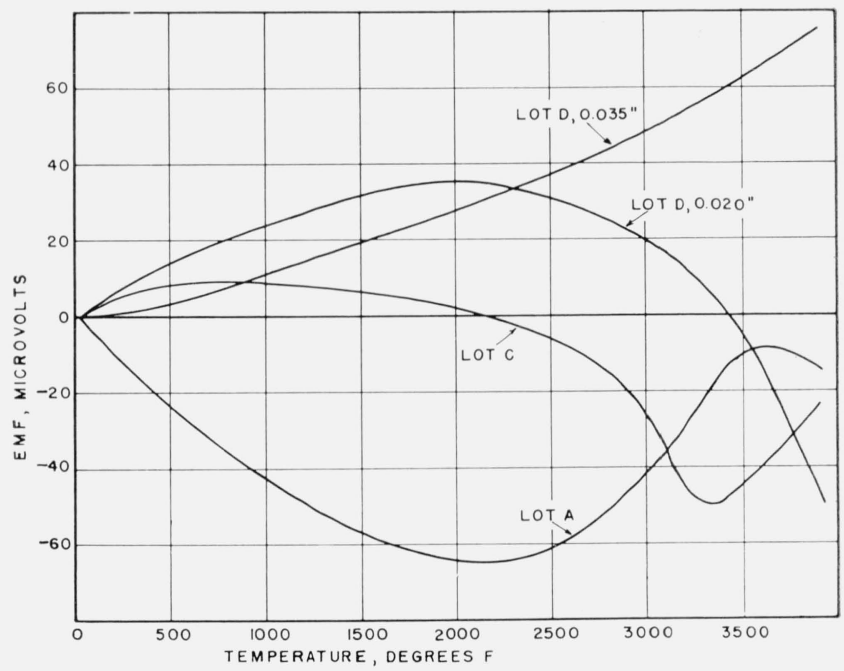

Figure 4. Emf differences between the lots of 40 percent rhodium and the corresponding table values.

(Thermocouple minus table values.)

the difference observed earlier for the 60 percent $\mathrm{Rh}$, and is not considered unduly large. The emf differences for the four 40 percent $\mathrm{Rh}$ lots are plotted in figure 4. For comparison of thermal emfs from other sources, table 3 gives emfs from the present work and those of Zysk [4], and of Sine [5] as reported by Caldwell [6]. The largest difference is about 250 $\mu \mathrm{v}$ at approximately $1800^{\circ} \mathrm{F}$ between Sine's and the NBS values. This corresponds to a temperature difference of about $80^{\circ} \mathrm{F}$, which is about twice the difference found between lots at NBS. 
TABLE 3. Emf of 60 percent Ir-40 percent Rh versus Ir thermocouples

\begin{tabular}{|c|c|c|c|}
\hline Temperature & NBS & Zysk & Sine \\
\hline $\begin{array}{l}{ }^{\circ} F \\
200 \\
400 \\
600 \\
800 \\
1000\end{array}$ & $\begin{array}{r}\text { Millivolts } \\
0.342 \\
.864 \\
1.468 \\
2.119 \\
2.792\end{array}$ & \begin{tabular}{r|} 
Millivolts \\
2.101 \\
2.764
\end{tabular} & $\begin{array}{r}\text { Millivolts } \\
0.32 \\
.82 \\
1.39 \\
2.00 \\
2.62\end{array}$ \\
\hline $\begin{array}{l}1200 \\
1400 \\
1600 \\
18000\end{array}$ & $\begin{array}{l}\text { 3. } 469 \\
4.131 \\
4.775 \\
5.397 \\
6.000\end{array}$ & $\begin{array}{l}3.433 \\
4.102 \\
4.741 \\
5.357 \\
5.956\end{array}$ & $\begin{array}{l}\text { 3. } 26 \\
3.90 \\
4.53 \\
5.15 \\
5.76\end{array}$ \\
\hline $\begin{array}{l}2200 \\
2400 \\
2600 \\
2800 \\
3000\end{array}$ & $\begin{array}{l}6.586 \\
7.159 \\
7.726 \\
8.291 \\
8.862\end{array}$ & $\begin{array}{l}6.540 \\
7.106 \\
-0\end{array}$ & $\begin{array}{l}6.36 \\
6.95 \\
7.54 \\
8.14 \\
8.77\end{array}$ \\
\hline $\begin{array}{l}3200 \\
3400 \\
3600 \\
3800\end{array}$ & $\begin{array}{r}9.444 \\
10.040 \\
10.653 \\
11.284\end{array}$ & 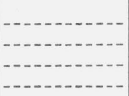 & $\begin{array}{r}9.42 \\
10.09 \\
10.78 \\
-.-2\end{array}$ \\
\hline
\end{tabular}

For the 50 percent $\mathrm{Rh}$ thermocouple the maximum difference between lots was about $80 \mu \mathrm{v}$. The deviations from the table values were more regular than those for the 40 percent $\mathrm{Rh}$ thermocouples and increased to as much as $40 \mu \mathrm{v}$ at $3900{ }^{\circ} \mathrm{F}$. The deviations of lot $\mathrm{A}$ and those of the 0.030 -in. wire of lot $\mathrm{F}$ were numerically about equal, and of opposite sign, while the emf of 0.020 -in. wire of lot $\mathrm{G}$ was within $6 \mu \mathrm{v}$ of the table values throughout the range.

As has been mentioned in a previous section and indicated in table 1 , the alloys $10,25,75$, and 90 percent $\mathrm{Rh}$ were each represented by two lots of wire. The maximum differences between their thermal emfs and the equivalent temperature differences are listed in table 4 . For the 10 and 25 percent $\mathrm{Rh}$ the maximum differences occurred at a temperature under $2000{ }^{\circ} \mathrm{F}$, lot A producing a higher output than lot $\mathrm{G}$. With increasing temperature the differences diminished, until equal outputs were obtained from the two lots, at about $3350^{\circ} \mathrm{F}$ for both compositions. For the 75 and 90 percent $\mathrm{Rh}$ the emf of lot $\mathrm{G}$ was lower than that of lot $\mathrm{A}$ at all temperatures, as indicated by the negative signs in table 4 .

TABLE 4. Maximum difference in thermal emf between thermocouples of lots $A$ and $G$

\begin{tabular}{|c|c|c|c|c|}
\hline & \multicolumn{4}{|c|}{ Percent $\mathrm{Rh}$} \\
\hline & 10 & 25 & 75 & 90 \\
\hline $\begin{array}{l}\text { Maximum difference } \\
\text { Equivalent temp, difference }\end{array}$ & $\begin{array}{l}236 \\
166\end{array}$ & $\begin{array}{r}108 \\
42\end{array}$ & $\begin{array}{r}-180 \\
-52\end{array}$ & $\begin{array}{r}-195 \\
-65\end{array}$ \\
\hline Temperature at maximum difference & 1900 & 1700 & 3700 & 3500 \\
\hline
\end{tabular}

The output of 40 percent $\mathrm{Rh}$ is the same as that of the 60 percent $\mathrm{Rh}$ at about $3000^{\circ} \mathrm{F}$. Below that temperature the 40 percent $\mathrm{Rh}$ has a higher emf than the 60 percent $\mathrm{Rh}$; above $3000^{\circ} \mathrm{F}$ its emf is less.
As the slopes of the emf-temperature curves for these two alloys do not differ greatly, the temperature at which equal emfs are observed may vary considerably from one thermocouple to another of the same nominal composition.

As was noted in the discussion of the work on the 60 percent $R h$ [1], several sources of error make increasingly large contributions to the uncertainty of the emfs obtained at temperatures above about $3500{ }^{\circ} \mathrm{F}$. They combine to produce an uncertainty estimated not to exceed $\pm 12{ }^{\circ} \mathrm{F}$ at the highest temperature.

The alloys of most interest from the standpoint of usefulness are the 40,50 , and 60 percent $\mathrm{Rh}$. While the present work has not yielded results on which a definite preference can be based, the 50 percent $\mathrm{Rh}$ would seem to be a logical choice, inasmuch as its emf is higher than that of either the 40 or 60 percent $\mathrm{Rh}$. If conditions of use were such as to bring about a change in composition at and near the measuring junction, as for example by preferential volatilization of one of the constituents of the alloy leg, the thermal emf would be changed relatively little over a range of several percent change of composition in the 50 percent $\mathrm{Rh}$ thermocouple. It is quite likely that extensive use of these thermocouples in different environments will indicate the superiority of one alloy over another, or different alloys may be best suited for different purposes. Even the alloys having less than 40 and more than 60 percent rhodium may have desirable properties.

The question of tolerances on composition has not been considered in this work, since the behavior of the thermocouples has indicated that other factors, some of them not identifiable, have predominated in causing differences or changes in thermocouple output.

It can be concluded from this study that a thermocouple of nominal composition 40,50 , or 60 percent $\mathrm{Rh}$ versus Ir can be used for measuring temperatures up to $3900{ }^{\circ} \mathrm{F}^{5}$ to an accuracy of $\pm 40{ }^{\circ} \mathrm{F}$ if the temperature of the thermocouple is determined from. the appropriate table. Higher accuracy can be achieved by calibrating the particular thermocouple in question. A convenient method of performing such a calibration is to measure the emf of each thermoelement of the test thermocouple against the corresponding element of a thermocouple of known thermal emf. A higher degree of accuracy can be obtained above $1900{ }^{\circ} \mathrm{F}$ by using an optical pyrometer and a blackbody with the method described earlier. If the emf of a thermocouple is to be determined at temperatures between calibration points, a curve of deviations from the appropriate reference table can be constructed and used as described in reference 7 .

${ }_{5}$ The temperature versus emf tables for the 60 percent $\mathrm{Rh}$ thermocouple extends to $3800{ }^{\circ} \mathrm{F}$. 


\section{Appendix}

TABLE 1-A. Sixty percent iridium-forty percent rhodium versus iridium thermocouples

Filectromotive force in absolute millivolts. Temperatures in degrees C (Int. 1948). Reference junctions at $0{ }^{\circ} \mathrm{C}$.

\begin{tabular}{|c|c|c|c|c|c|c|c|c|c|c|c|c|}
\hline \multirow{2}{*}{$\begin{array}{l}\text { Milli- } \\
\text { volts }\end{array}$} & .000 & .010 & .020 & .030 & .040 & .050 & .060 & .070 & .080 & .090 & .100 & Milli- \\
\hline & \multicolumn{12}{|c|}{ Degrees C } \\
\hline 0.000 & 0.0 & 3.2 & 6.4 & 9.5 & 12.6 & 15.6 & 18.7 & 21.6 & 24.6 & 27.5 & 30.4 & 0.000 \\
\hline 0.100 & 30.4 & 33.2 & 36.1 & 38.9 & 41.6 & 44.4 & 47.1 & 49.8 & 52.5 & 55.1 & 57.8 & 0.100 \\
\hline 0.200 & 57.8 & 60.4 & 63.0 & 65.5 & 68.1 & 70.6 & 73.2 & 75.7 & 78.1 & 80.6 & 83.1 & 0.200 \\
\hline 0.300 & 83.1 & 85.5 & 87.9 & 90.3 & 92.7 & 95.1 & 97.5 & 99.8 & 102.2 & 104.5 & 106.8 & 0.300 \\
\hline 0.400 & 106.8 & 109.1 & 111.4 & 113.7 & 116.0 & 118.2 & 120.5 & 122.7 & 124.9 & 127.1 & 129.4 & 0.400 \\
\hline 0.500 & 129.4 & 131.6 & 133.8 & 135.9 & 138.1 & 140.3 & 142.4 & 144.6 & 146.7 & 148.8 & 151.0 & 0.500 \\
\hline 0.600 & 151.0 & 153.1 & 155.2 & 157.3 & 159.4 & 161.5 & 163.5 & 165.6 & 167.7 & 169.7 & 171.8 & 0.600 \\
\hline 0.700 & 171.8 & 173.8 & 175.8 & 177.9 & 179.9 & 181.9 & 183.9 & 185.9 & 187.9 & 189.9 & 191.9 & 0.700 \\
\hline 0.800 & 191.9 & 193.9 & 195.9 & 197.9 & 199.8 & 20 r. 8 & 203.7 & 205.7 & 207.6 & 209.6 & 211.5 & 0.800 \\
\hline 0.900 & 211.5 & 213.4 & 215.4 & 217.3 & 219.2 & 221.1 & 223.0 & 224.9 & 226.8 & 228.7 & 230.6 & 0.900 \\
\hline 1.000 & 230.6 & 232.5 & 234.4 & 236.3 & 238.2 & 240.1 & 241.9 & 243.8 & 245.7 & 247.5 & 249.4 & 1.000 \\
\hline 1.100 & 249.4 & 251.2 & 253.1 & 254.9 & 256.8 & 258.6 & 260.4 & 262.3 & 264.1 & 265.9 & 267.7 & 1.100 \\
\hline 1.200 & 267.7 & 269.6 & 271.4 & 273.2 & 275.0 & 276.8 & 278.6 & 280.4 & 282.2 & 284.0 & 285.8 & 1.200 \\
\hline 1.300 & 285.8 & 287.6 & 289.4 & 291.2 & 293.0 & 294.8 & 296.6 & 298.3 & 300.1 & 301.9 & 303.6 & 1.300 \\
\hline 1.400 & 303.6 & 305.4 & 307.2 & 308.9 & 310.7 & 312.5 & 314.2 & 316.0 & 317.7 & 319.5 & 321.2 & 1.400 \\
\hline 1.500 & 321.2 & 323.0 & 324.7 & 326.5 & 328.2 & 329.9 & 331.7 & 333.4 & 335.2 & 336.9 & 338.6 & 1.500 \\
\hline 1.600 & 338.6 & 340.3 & 342.1 & 343.8 & 345.5 & 347.2 & 349.0 & 350.7 & 352.4 & 354.1 & 355.8 & 1.600 \\
\hline 1.700 & 355.8 & 357.6 & 359.3 & 361.0 & 362.7 & 364.4 & 366.1 & 367.8 & 369.5 & 371.2 & 372.9 & 1.700 \\
\hline 1.800 & 372.9 & 374.6 & 376.3 & 378.0 & 379.7 & 381.4 & 383.1 & 384.8 & 386.5 & 388.2 & 389.8 & 1.800 \\
\hline 1.900 & 389.8 & 391.5 & 393.2 & 394.9 & 396.6 & 398.3 & 399.9 & 401.6 & 403.3 & 405.0 & 406.7 & 1.900 \\
\hline 2.000 & 406.7 & 408.4 & 410.0 & 411.7 & 413.4 & 415.1 & 416.7 & 418.4 & $420.1^{\circ}$ & 421.7 & 423.4 & 2.000 \\
\hline 2.100 & 423.4 & 425.1 & 426.7 & 428.4 & 430.1 & 431.7 & 433.4 & 435.1 & 436.7 & 438.4 & 440.1 & 2.100 \\
\hline 2.200 & 440.1 & 441.7 & 443.4 & 445.1 & 446.7 & 448.4 & 450.0 & 451.7 & 453.4 & 455.0 & 456.7 & 2.200 \\
\hline 2.300 & 456.7 & 458.3 & 460.0 & 461.6 & 463.3 & 464.9 & 466.6 & 468.2 & 469.9 & 471.6 & 473.2 & 2.300 \\
\hline 2.400 & 473.2 & 474.9 & 476.5 & 478.2 & 479.8 & 481.5 & 483.1 & 484.8 & 486.4 & 488.1 & 489.7 & 2.400 \\
\hline 2.500 & 489.7 & 491.4 & 493.0 & 494.6 & 496.3 & 497.9 & 499.6 & 501.2 & 502.9 & 504.5 & 506.2 & 2.500 \\
\hline 2.600 & 506.2 & 507.8 & 509.5 & 511.1 & 512.8 & 514.4 & 516.1 & 517.7 & 519.3 & 521.0 & 522.6 & 2.600 \\
\hline 2.700 & 522.6 & 524.3 & 525.9 & 527.6 & 529.2 & 530.8 & 532.5 & 534.1 & 535.8 & 537.4 & 539.1 & 2.700 \\
\hline 2.800 & 539.1 & 540.7 & 542.3 & 544.0 & 545.6 & 547.2 & 548.8 & 550.5 & 552.1 & 553.7 & 555.4 & 2.800 \\
\hline 2.900 & 555.4 & 557.0 & 558.6 & 560.3 & 561.9 & 563.5 & 565.2 & 566.8 & 568.4 & 570.1 & 571.7 & 2.900 \\
\hline 3.000 & 571.7 & 573.4 & 575.0 & 576.6 & 578.3 & 579.9 & 581.5 & 583.2 & 584.8 & 586.5 & 588.1 & 3.000 \\
\hline 3.100 & 588.1 & 589.7 & 591.4 & 593.0 & 594.7 & 596.3 & 597.9 & 599.6 & 601.2 & 602.9 & 604.5 & 3.100 \\
\hline 3.200 & 604.5 & 606.1 & 607.8 & 609.4 & 611.1 & 612.7 & 614.4 & 616.0 & 617.7 & 619.3 & 621.0 & 3.200 \\
\hline 3.300 & 621.0 & 622.6 & 624.3 & 625.9 & 627.6 & 629.2 & 630.9 & 632.5 & 634.2 & 635.8 & 637.5 & 3.300 \\
\hline 3.400 & 637.5 & 639.1 & 640.8 & 642.4 & 644.1 & 645.7 & 647.4 & 649.1 & 650.7 & 652.4 & 654.0 & 3.400 \\
\hline 3.500 & 654.0 & 655.7 & 657.3 & 659.0 & 660.7 & 662.3 & 664.0 & 665.6 & 667.3 & 669.0 & 670.6 & 3.500 \\
\hline 3.600 & 670.6 & 672.3 & 674.0 & 675.6 & 677.3 & 679.0 & 680.6 & 682.3 & 684.0 & 685.6 & 687.3 & 3.600 \\
\hline 3.700 & 687.3 & 689.0 & 690.7 & 692.3 & 694.0 & 695.7 & 697.3 & 699.0 & 700.7 & 702.4 & 704.0 & 3.700 \\
\hline 3.800 & 704.0 & 705.7 & 707.4 & 709.1 & 710.8 & 712.4 & 714.1 & 715.8 & 717.5 & 719.2 & 720.8 & 3.800 \\
\hline 3.900 & 720.8 & 722.5 & 724.2 & 725.9 & 727.6 & 729.3 & 730.9 & 732.6 & 734.3 & 736.0 & 737.7 & 3.900 \\
\hline 4.000 & 737.7 & 739.4 & 741.1 & 742.8 & 744.5 & 746.2 & 747.9 & 749.6 & 751.3 & 753.0 & 754.6 & 4.000 \\
\hline 4.100 & 754.6 & 756.3 & 758.0 & 759.7 & 761.4 & 763.1 & 764.9 & 766.6 & 768.3 & 770.0 & 771.7 & 4.100 \\
\hline 4.200 & 771.7 & 773.4 & 775.1 & 776.8 & 788.5 & 780.2 & 781.9 & 783.6 & 785.3 & 787.1 & 788.8 & 4.200 \\
\hline 4.300 & 788.8 & 790.5 & 792.2 & 793.9 & 795.6 & 797.4 & 799.1 & 800.8 & 802.5 & 804.2 & 806.0 & 4.300 \\
\hline 4.400 & 806.0 & 807.7 & 809.4 & 811.1 & 812.8 & 814.6 & 816.3 & 818.0 & 819.8 & 821.5 & 823.2 & 4.400 \\
\hline 4.500 & 823.2 & 824.9 & 826.7 & 828.4 & 830.1 & 831.9 & 833.6 & 835.3 & 837.1 & 838.8 & 840.6 & 4.500 \\
\hline 4.600 & 840.6 & 842.3 & 844.0 & 845.8 & 847.5 & 849.3 & 851.0 & 852.8 & 854.5 & 856.3 & 858.0 & 4.600 \\
\hline 4.700 & 858.0 & 859.8 & 861.5 & 863.3 & 865.0 & 866.8 & 868.5 & 870.3 & 872.0 & 873.8 & 875.5 & 4.700 \\
\hline 4.800 & 875.5 & 877.3 & 879.0 & 880.8 & 882.6 & 884.3 & 886.1 & 887.9 & 889.6 & 891.4 & 893.2 & 4.800 \\
\hline 4.900 & 893.2 & 894.9 & 896.7 & 898.5 & 900.2 & 902.0 & 903.8 & 905.5 & 907.3 & 909.1 & 910.9 & 4.900 \\
\hline
\end{tabular}


TABLE 1-A. Sixty percent iridium-forty percent rhodium versus iridium thermocouples-Continued

Electromotive force in absolute millivolts. Temperatures in degrees C (Int. 1948). Reference junctions at $0{ }^{\circ} \mathrm{C}$.

\begin{tabular}{|c|c|c|c|c|c|c|c|c|c|c|c|c|}
\hline \multirow{2}{*}{$\begin{array}{l}\text { Milli- } \\
\text { volts }\end{array}$} & .000 & .010 & .020 & .030 & .040 & .050 & .060 & .070 & .080 & .090 & .100 & \multirow{2}{*}{$\begin{array}{l}\text { Milli- } \\
\text { volts }\end{array}$} \\
\hline & \multicolumn{11}{|c|}{ Degrees C } & \\
\hline 000 & 910.9 & 912.6 & 914.4 & 916.2 & 918.0 & 919.8 & 921.5 & 923.3 & 925.1 & 926.9 & 928.7 & 5.000 \\
\hline 100 & 928.7 & 930.5 & 932.3 & 934.0 & 935.8 & 937.6 & 939.4 & 941.2 & 943.0 & 944.8 & 946.6 & 5.100 \\
\hline 5.200 & 946.6 & 948.4 & 950.2 & 952.0 & 953.8 & 955.6 & & & & & 964.6 & 5.200 \\
\hline 5.300 & 964.6 & 966.4 & 968.2 & 970.0 & 971.8 & 973.6 & 975.4 & 977.3 & 979.1 & 980.9 & 982.7 & 5.300 \\
\hline 5.400 & 982.7 & 984.5 & 986.3 & 988.1 & 990.0 & 991.8 & 993.6 & 995.4 & 997.2 & 999.1 & 1000.9 & 5.400 \\
\hline 5.500 & 1000.9 & 1002.7 & 1004.5 & 1006.4 & 1008.2 & 1010.0 & 1011.9 & 1013.7 & 1015.5 & 1017.4 & 1019.2 & .500 \\
\hline 5.600 & 1019.2 & 1021.0 & 1022.9 & 1024.7 & 1026.5 & 1028.4 & 1030.2 & 1032.1 & 103 & 1035.7 & 1037.6 & .600 \\
\hline 5.700 & 1037.6 & 1039.4 & 1041.3 & 1043.1 & 1045.0 & 1046.8 & 1048.7 & 1050.5 & 1052.4 & 1054.2 & 1056.1 & 5.700 \\
\hline 5.800 & 1056.1 & 1057.9 & 1059.8 & 1061.6 & 1063.5 & 1065.3 & 1067.2 & 1069.1 & 1070.9 & 1072.8 & 1074.7 & 5.800 \\
\hline 5.900 & 1074.7 & 1076.5 & 1078.4 & 1080.2 & 1082.1 & 1084.0 & 1085.8 & 1087.7 & 1089.6 & 1091.5 & 1093.3 & 5.900 \\
\hline 6.000 & 93.3 & 5.2 & & & 00.8 & & & & & & & 000 \\
\hline 6.100 & & & & & .6 & & & & & & & 100 \\
\hline 6.200 & 1130.9 & 1132.8 & 1134.7 & 1136.6 & 1138.5 & 1140.4 & & 1144.2 & 1146.1 & & & 6.200 \\
\hline 6.300 & 1149.9 & 1151.8 & 1153.7 & 1155.6 & 1157.5 & 1159.4 & 1161.3 & 1163.2 & 1165.1 & 1167.0 & 1168.9 & 6.300 \\
\hline 6.400 & 1168.9 & 1170.8 & 1172.7 & & 1176.5 & & & & & & & .400 \\
\hline 6.500 & 1188.0 & 1189.9 & 11 & 3.8 & 7 & 119 & & & & & & 500 \\
\hline 6.600 & 1207.2 & 1209.1 & & & 9 & & & & & & & .600 \\
\hline 6.700 & 1226.4 & 1228.4 & 1230.3 & 1232.2 & 1234.1 & 1236 & 1238.0 & 1239.9 & 124 & & & 6.700 \\
\hline 6.800 & 1245.7 & 1247.7 & 1249.6 & 1251.5 & 1253.5 & 1255.4 & 1257.3 & 1259.3 & 1261.2 & 1263.2 & 1265.1 & 6.800 \\
\hline 6.900 & 1265.1 & 1267.0 & 1269.0 & 1270.9 & 1272.9 & 1274.8 & 1276.8 & 1278.7 & 1280.6 & 1282.6 & 1284.5 & 6.900 \\
\hline 7.000 & & & & & & & & & & & & \\
\hline 7.100 & 1304.0 & 1306.0 & & & 1311.8 & $131:$ & & & & & & 7.100 \\
\hline 7.200 & 1323.5 & 1325.5 & 1327.4 & 1329.4 & 1331.3 & 133 & & 1337.2 & & & 1343.1 & 7.200 \\
\hline 7.300 & 1343.1 & & & & & & & & & & & 7.300 \\
\hline 7.400 & 52.7 & 4.7 & & & .5 & & & & & & & 100 \\
\hline 7.500 & 1382.3 & 1384.3 & 1386.2 & 8.2 & & & & & & & & 7.500 \\
\hline 7.600 & 1402.0 & 1403.9 & & & 1409.8 & & & & & & & 7.600 \\
\hline 7.700 & 1421.6 & 1423.6 & 1425.5 & 1427.5 & 1429.5 & 1431.4 & & 1435.4 & & 1439.3 & & 7.700 \\
\hline 7.800 & 1441.3 & 1443.2 & & & & & & & & & & 7.800 \\
\hline 7.900 & 1460.9 & 1462.9 & 1464.9 & 1466.8 & 1468.8 & 1470.8 & 1472.7 & 1474.7 & 1476.7 & 1478.6 & 1480.6 & 7.900 \\
\hline 8.000 & & & & & & & & & & & & \\
\hline 8.100 & 1500.2 & 1502.2 & 1504.2 & 1506.1 & 1508.1 & & & & & & & 8.100 \\
\hline 8.200 & & & & & & & & & & & & 8.200 \\
\hline 8.300 & & & & & & & & & & & & .300 \\
\hline 8.400 & & & & 4.9 & 6.8 & & & & & & & 3.400 \\
\hline 8.500 & 8.5 & 30.5 & & 34.4 & 36.3 & & & & & & & 3.500 \\
\hline 8.600 & 1598.0 & 1600.0 & 1601.9 & 1603.9 & 1605.8 & 1607.7 & & 1611.6 & & & 1617.5 & 8.600 \\
\hline 8.700 & 1617.5 & & & & & & & & & & & 8.700 \\
\hline 8.800 & & & & & & & & & & & & 8.800 \\
\hline 8.900 & 1656.2 & 1658.1 & 1660.0 & 1661.9 & 1663.9 & 1665.8 & 1667.7 & 1669.7 & 1671.6 & 1673.5 & 1675.4 & 8.900 \\
\hline 9.000 & & & & & & & & & & & & \\
\hline 9.100 & 1694.6 & 1696.5 & & & & & & & & & & 9.100 \\
\hline 9.200 & 1713.7 & & & & & & & & & & & 9.200 \\
\hline 9.300 & & & & & & & & & & & & \\
\hline 9.400 & 1751.7 & 1753.6 & & & 59.3 & & & & & & 1770.6 & 9.400 \\
\hline 9.500 & 1770.6 & 1772.5 & & & & & & & & & & 9.500 \\
\hline 9.600 & 1789.4 & 1791.3 & 1793.2 & 1795.1 & 1796.9 & 1798.8 & 1800.7 & 1802.5 & 1804.4 & 1806.3 & 1808.2 & 9.600 \\
\hline 9.700 & 1808.2 & 1810.0 & 1811.9 & 1813.8 & 1815.6 & 1817.5 & 1819.3 & 1821.2 & 1823.1 & 1824.9 & 1826.8 & 9.700 \\
\hline 9.800 & 1826.8 & 1828.6 & & & 1834.2 & & & 1839.8 & & & 1845.3 & 9.800 \\
\hline 9.900 & 1845.3 & 1847.2 & 1849.0 & 1850.9 & 1852.7 & 1854.6 & 1856.4 & 1858.3 & 1860.1 & 1862.0 & 1863.8 & 9.900 \\
\hline
\end{tabular}


TABLE 1-A. Sixty percent iridium-forty percent rhodium versus iridium thermocouples-Continued

Electromotive force in absolute millivolts. Temperatures in degrees $\mathrm{C}$ (Int. 1948). Reference junctions at $0^{\circ} \mathrm{C}$.

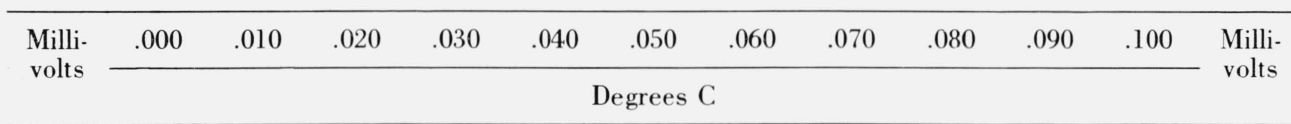

\begin{tabular}{|c|c|c|c|c|c|c|c|c|c|c|c|c|}
\hline 10.000 & 1863.8 & 1865.6 & 1867.5 & 1869.3 & 1871.2 & 1873.0 & 1874 & 1876 & 1878 & 1880.3 & & \\
\hline & 1882.2 & 1884.0 & 1885.8 & 1887.7 & 1889.5 & 1891.3 & 1893.1 & 1895.0 & 1896.8 & 1898.6 & 1900. & \\
\hline 0.300 & 1918.6 & 920.5 & 1922.3 & 1924.1 & 1925.9 & 1927.7 & 1929.5 & 31.3 & 1933.1 & 1934.9 & 936.7 & \\
\hline 0.500 & 954.8 & 1956.6 & 1958.4 & 1960.2 & 1962.0 & 1963.7 & 1965 & 1967.3 & 1969.1 & 1970.9 & 972.7 & \\
\hline 0.600 & 1972.7 & 1974.5 & 76.3 & & & & & & & & & \\
\hline 0.700 & 1990.6 & 1992.4 & 1994.1 & 1995.9 & & 1999.5 & 200 & 20 & & 2006.6 & 3 & \\
\hline & 2026.0 & 2027.8 & 2029.6 & 2031.3 & 2033.1 & 2034.8 & 2036.6 & 2038.4 & 2040.1 & & & \\
\hline 0 & & & & & & & & & & & & \\
\hline 1.100 & 2061.2 & 2062.9 & 2064.7 & 2066.4 & & 2069.9 & 20 & & 20 & & & \\
\hline 1.200 & 2078.6 & 2080.4 & 2082.1 & 2083.9 & 2085 & 2087.4 & 208 & & 2092 & 209 & 20 & 1. \\
\hline 11.300 & 2096.0 & 2097.8 & 2099.5 & 2101.2 & 2103.0 & 2104.7 & 2106 & & & & & \\
\hline
\end{tabular}

TABLE 2-A. Sixty percent iridium-forty percent rhodium versus iridium thermocouples

\begin{tabular}{|c|c|c|c|c|c|c|c|c|c|c|c|c|}
\hline \multirow{2}{*}{${ }^{\circ} \mathrm{C}$} & 0 & 10 & 20 & 30 & 40 & 50 & 60 & 70 & 80 & 90 & 100 & \multirow{2}{*}{${ }^{\circ} \mathrm{C}$} \\
\hline & \multicolumn{11}{|c|}{ Millivolts } & \\
\hline 0 & 0.000 & 0.032 & 0.064 & 0.099 & 0.134 & 0.171 & 0.209 & 0.247 & 0.287 & 0.329 & 0.371 & \\
\hline 100 & 0.371 & 0.414 & 0.458 & 0.503 & 0.549 & 0.595 & 0.643 & 0.691 & 0.740 & 0.790 & 0.841 & 100 \\
\hline 200 & 0.841 & 0.892 & 0.944 & 0.997 & 1.050 & 1.103 & 1.158 & 1.212 & 1.268 & 1.323 & 1.379 & 200 \\
\hline 300 & 1.379 & 1.436 & 1.493 & 1.550 & 1.608 & 1.666 & 1.724 & 1.783 & 1.842 & 1.901 & 1.960 & 300 \\
\hline 400 & 1.960 & 2.020 & 2.080 & 2.139 & 2.200 & 2.260 & 2.320 & 2.381 & 2.441 & 2.502 & 2.562 & 400 \\
\hline 500 & 2.562 & 2.623 & 2.684 & 2.745 & 2.806 & 2.867 & 2.928 & 2.989 & 3.051 & 3.112 & 3.173 & 500 \\
\hline 600 & 3.173 & 3.233 & 3.294 & 3.355 & 3.415 & 3.476 & 3.536 & 3.596 & 3.656 & 3.716 & 3.776 & 600 \\
\hline 700 & 3.776 & 3.835 & 3.895 & 3.954 & 4.014 & 4.073 & 4.131 & 4.190 & 4.249 & 4.307 & 4.365 & 700 \\
\hline 800 & 4.365 & 4.423 & 4.481 & 4.539 & 4.597 & 4.654 & 4.711 & 4.768 & 4.825 & 4.882 & 4.939 & 800 \\
\hline 900 & 4.939 & 4.995 & 5.051 & 5.107 & 5.163 & 5.219 & 5.274 & 5.330 & 5.385 & 5.440 & 5.495 & 900 \\
\hline 1000 & 5.495 & 5.550 & 5.604 & 5.659 & 5.713 & 5.76 & 5.82 & 5.875 & 5.9 & 5.9 & & 1000 \\
\hline 1100 & 6.036 & 6.089 & 6.142 & 6.195 & 6.248 & 6.301 & 6.353 & 6.406 & 6.458 & 6.510 & 6.5 & 1100 \\
\hline 1200 & 6.563 & 6.615 & 6.667 & 6.718 & 6.770 & 6.822 & 6.874 & 6.925 & 6.977 & 7.028 & 7.079 & 1200 \\
\hline 1300 & 7.079 & 7.131 & 7.182 & 7.233 & 7.284 & 7.335 & 7.386 & 7.437 & 7.488 & 7.539 & 7.590 & 1300 \\
\hline 1400 & 7.590 & 7.641 & 7.692 & 7.743 & 7.793 & 7.844 & 7.895 & 7.946 & 7.997 & 8.048 & 8.099 & 1400 \\
\hline 1500 & 8.099 & 8.150 & 8.201 & 8.252 & 8.303 & 8.354 & 8.405 & 8.456 & 8.507 & 8.559 & 8.610 & 1500 \\
\hline 1600 & 8.610 & 8.662 & 8.713 & 8.765 & 8.816 & 8.868 & 8.920 & 8.972 & 9.024 & 9.076 & 9.128 & 1600 \\
\hline 1700 & 9.128 & 9.180 & 9.233 & 9.285 & 9.338 & 9.391 & 9.444 & 9.497 & 9.550 & 9.603 & 9.656 & 1700 \\
\hline 1800 & 9.656 & 9.710 & 9.763 & 9.817 & 9.871 & 9.925 & 9.979 & 10.034 & 10.088 & 10.143 & 10.198 & 1800 \\
\hline 1900 . & 10.198 & 10.252 & 10.307 & 10.363 & 10.418 & 10.473 & 10.529 & 10.585 & 10.641 & 10.697 & 10.753 & 1900 \\
\hline 2000 & & & & 10.922 & 10.979 & 11.036 & 11.093 & 11.150 & 11.208 & 11.265 & 11.323 & 2000 \\
\hline 2100 & 11.323 & 11.380 & 11.438 & 11.496 & 11.554 & 11.612 & & & & & & 2100 \\
\hline
\end{tabular}


TABLE 3-A. Sixty percent iridium-forty percent rhodium versus iridium thermocouples

Electromotive force in absolute millivolts. Temperatures in degrees $\mathrm{F}^{*}$. Reference junctions at $32^{\circ} \mathrm{F}$.

\begin{tabular}{|c|c|c|c|c|c|c|c|c|c|c|c|c|}
\hline \multirow{2}{*}{$\begin{array}{l}\text { Milli- } \\
\text { volts }\end{array}$} & .000 & .010 & .020 & .030 & .040 & .050 & .060 & .070 & .080 & .090 & .100 & \multirow{2}{*}{$\begin{array}{l}\text { Milli- } \\
\text { volts }\end{array}$} \\
\hline & \multicolumn{11}{|c|}{ Degrees F } & \\
\hline 0.000 & 32.0 & 37.8 & 43.5 & 49.1 & 54.7 & 60.2 & 65.6 & 70.9 & 76.2 & 81.5 & 86.7 & 0.000 \\
\hline 0.100 & 86.7 & 91.8 & 96.9 & 101.9 & 106.9 & 111.9 & 116.7 & 121.6 & 126.5 & 131.2 & 136.0 & 0.100 \\
\hline 0.200 & 136.0 & 140.7 & 145.3 & 150.0 & 154.6 & 159.1 & 163.7 & 168.2 & 172.6 & 177.1 & 181.5 & 0.200 \\
\hline 0.300 & 181.5 & 185.9 & 190.3 & 194.6 & 198.9 & 203.2 & 207.5 & 211.7 & 215.9 & 220.1 & 224.3 & 0.300 \\
\hline 0.400 & 224.3 & 228.4 & 232.5 & 236.6 & 240.7 & 244.8 & 248.9 & 252.9 & 256.9 & 260.9 & 264.9 & 0.400 \\
\hline 0.500 & 264.9 & 268.8 & 272.8 & 276.7 & 280.6 & 284.5 & 288.4 & 292.2 & 296.1 & 299.9 & 303.7 & 0.500 \\
\hline 0.600 & 303.7 & 307.5 & 311.3 & 315.1 & 318.9 & 322.6 & 326.4 & 330.1 & 333.8 & 337.5 & 341.2 & 0.600 \\
\hline 0.700 & 341.2 & 344.9 & 348.5 & 352.2 & 355.8 & 359.5 & 363.1 & 366.7 & 370.3 & 373.9 & 377.5 & 0.700 \\
\hline 0.800 & 377.5 & 381.0 & 384.6 & 388.1 & 391.7 & 395.2 & 398.7 & 402.2 & 405.7 & 409.2 & 412.7 & 0.800 \\
\hline 0.900 & 412.7 & 416.2 & 419.7 & 423.1 & 426.6 & 430.0 & 433.5 & 436.9 & 440.3 & 443.8 & 447.2 & 0.900 \\
\hline 1.000 & 447.2 & 450.6 & 453.9 & 457.3 & 460.7 & 464.1 & 467.5 & 470.8 & 474.2 & 477.5 & 480.9 & 1.000 . \\
\hline 1.100 & 480.9 & 484.2 & 487.5 & 490.9 & 494.2 & 497.5 & 500.8 & 504.1 & 507.4 & 510.7 & 513.9 & 1.100 \\
\hline 1.200 & 513.9 & 517.2 & 520.5 & 523.8 & 527.0 & 530.3 & 533.5 & 536.8 & 540.0 & 543.3 & 546.5 & 1.200 \\
\hline 1.300 & 546.5 & 549.7 & 552.9 & 556.1 & 559.4 & 562.6 & 565.8 & 569.0 & 572.2 & 575.4 & 578.6 & 1.300 \\
\hline 1.400 & 578.6 & 581.7 & 584.9 & 588.1 & 591.3 & 594.4 & 597.6 & 600.8 & 603.9 & 607.1 & 610.2 & 1.400 \\
\hline 1.500 & 610.2 & 613.4 & 616.5 & 619.7 & 622.8 & 625.9 & 629.0 & 632.2 & 635.3 & 638.4 & 641.5 & 1.500 \\
\hline 1.600 & 641.5 & 644.6 & 647.7 & 650.8 & 654.0 & 657.1 & 660.2 & 663.2 & 666.3 & 669.4 & 672.5 & 1.600 \\
\hline 1.700 & 672.5 & 675.6 & 678.7 & 681.8 & 684.8 & 687.9 & 691.0 & 694.1 & 697.1 & 700.2 & 703.2 & 1.700 \\
\hline 1.800 & 703.2 & 706.3 & 709.4 & 712.4 & 715.5 & 718.5 & 721.6 & 724.6 & 727.6 & 730.7 & 733.7 & 1.800 \\
\hline 1.900 & 733.7 & 736.8 & 739.8 & 742.8 & 745.9 & 748.9 & 751.9 & 754.9 & 758.0 & 761.0 & 764.0 & 1.900 \\
\hline 2.000 & 764.0 & 767.0 & 770.1 & 773.1 & 776.1 & 779.1 & 782.1 & 785.1 & 788.1 & 791.1 & 794.1 & 2.000 \\
\hline 2.100 & 794.1 & 797.1 & 800.2 & 803.2 & 806.2 & 809.2 & 812.2 & 815.1 & 818.1 & 821.1 & 824.1 & 2.100 \\
\hline 2.200 & 824.1 & 827.1 & 830.1 & 833.1 & 836.1 & 839.1 & 842.1 & 845.1 & 848.0 & 851.0 . & 854.0 & 2.200 \\
\hline 2.300 & 854.0 & 857.0 & 860.0 & 862.9 & 865.9 & 868.9 & 871.9 & 874.9 & 877.8 & 880.8 & 883.8 & 2.300 \\
\hline 2.400 & 883.8 & 886.7 & 889.7 & 892.7 & 895.7 & 898.6 & 901.6 & 904.6 & 907.5 & 910.5 & 913.5 & 2.400 \\
\hline 2.500 & 913.5 & 916.4 & 919.4 & 922.4 & 925.3 & 928.3 & 931.3 & 934.2 & 937.2 & 940.1 & 943.1 & 2.500 \\
\hline 2.600 & 943.1 & 946.1 & 949.0 & 952.0 & 955.0 & 957.9 & 960.9 & 963.8 & 966.8 & 969.8 & 972.7 & 2.600 \\
\hline 2.700 & 972.7 & 975.7 & 978.6 & 981.6 & 984.6 & 987.5 & 990.5 & 993.5 & 996.4 & 999.4 & 1002.3 & 2.700 \\
\hline 2.800 & 1002.3 & 1005.2 & 1008.2 & 1011.1 & 1014.0 & 1017.0 & 1019.9 & 1022.9 & 1025.8 & 1028.7 & 1031.7 & 2.800 \\
\hline 2.900 & 1031.7 & 1034.6 & 1037.6 & 1040.5 & 1043.4 & 1046.4 & 1049.3 & 1052.3 & 1055.2 & 1058.1 & 1061.1 & 2.900 \\
\hline 3.000 & 1061.1 & 1064.0 & 1067.0 & 1069.9 & 1072.9 & 1075.8 & 1078.8 & 1081.7 & 1084.7 & 1087.6 & 1090.6 & 3.000 \\
\hline 3.100 & 1090.6 & 1093.5 & 1096.5 & 1099.4 & 1102.4 & 1105.3 & 1108.3 & 1111.3 & 1114.2 & 1117.2 & 1120.1 & 3.100 \\
\hline 3.200 & 1120.1 & 1123.1 & 1126.0 & 1129.0 & 1131.9 & 1134.9 & 1137.9 & 1140.8 & 1143.8 & 1146.8 & 1149.7 & 3.200 \\
\hline 3.300 & 1149.7 & 1152.7 & 1155.7 & 1158.6 & 1161.6 & 1164.6 & 1167.5 & 1170.5 & 1173.5 & 1176.5 & 1179.4 & 3.300 \\
\hline 3.400 & 1179.4 & 1182.4 & 1185.4 & 1188.4 & 1191.4 & 1194.3 & 1197.3 & 1200.3 & 1203.3 & 1206.3 & 1209.3 & 3.400 \\
\hline 3.500 & 1209.3 & 1212.2 & 1215.2 & 1218.2 & 1221.2 & 1224.2 & 1227.2 & 1230.1 & 1233.1 & 1236.1 & 1239.1 & 3.500 \\
\hline 3.600 & 1239.1 & 1242.1 & 1245.1 & 1248.1 & 1251.1 & 1254.1 & 1257.1 & 1260.1 & 1263.1 & 1266.2 & 1269.2 & 3.600 \\
\hline 3.700 & 1269.2 & 1272.2 & 1275.2 & 1278.2 & 1281.2 & 1284.2 & 1287.2 & 1290.2 & 1293.2 & 1296.3 & 1293.3 & 3.700 \\
\hline 3.800 & 1299.3 & 1302.3 & 1305.3 & 1308.3 & 1311.4 & 1314.4 & 1317.4 & 1320.4 & 1323.5 & 1326.5 & 1329.5 & 3.800 \\
\hline 3.900 & 1329.5 & 1332.5 & 1335.6 & 1338.6 & 1341.6 & 1344.7 & 1347.7 & 1350.8 & 1353.8 & 1356.8 & 1359.9 & 3.900 \\
\hline 4.000 & 1359.9 & 1362.9 & 1366.0 & 1369.0 & 1372.1 & 1375.1 & 1378.2 & 1381.2 & 1384.3 & 1387.3 & 1390.4 & 4.000 \\
\hline 4.100 & 1390.4 & 1393.4 & 1396.5 & 1399.5 & 1402.6 & 1405.7 & 1408.7 & 1411.8 & 1414.9 & 1417.9 & 1421.0 & 4.100 \\
\hline 4.200 & 1421.0 & 1424.1 & 1427.2 & 1430.2 & 1433.3 & 1436.4 & 1439.5 & 1442.5 & 1445.6 & 1448.7 & 1451.8 & 4.200 \\
\hline 4.300 & 1451.8 & 1454.9 & 1458.0 & 1461.0 & 1464.1 & 1467.2 & 1470.3 & 1473.4 & 1476.5 & 1479.6 & 1482.7 & 4.300 \\
\hline 4.400 & 1482.7 & 1485.8 & 1488.9 & 1492.0 & 1495.1 & 1498.2 & 1501.3 & 1504.4 & 1507.5 & 1510.7 & 1513.8 & 4.400 \\
\hline 4.500 & 1513.8 & 1516.9 & 1520.0 & 1523.1 & 1526.2 & 1529.4 & 1532.5 & 1535.6 & 1538.7 & 1541.9 & 1545.0 & 4.500 \\
\hline 4.600 & 1545.0 & 1548.1 & 1551.3 & 1554.4 & 1557.6 & 1560.7 & 1563.8 & 1567.0 & 1570.1 & 1573.3 & 1576.4 & 4.600 \\
\hline 4.700 & 1576.4 & 1579.6 & 1582.7 & 1585.9 & 1589.0 & 1592.2 & 1595.3 & 1598.5 & 1601.6 & 1604.8 & 1608.0 & 4.700 \\
\hline 4.800 & 1608.0 & 1611.1 & 1614.3 & 1617.5 & 1620.6 & 1623.8 & 1627.0 & 1630.2 & 1633.3 & 1636.5 & 1639.7 & 4.800 \\
\hline 4.900 & 1639.7 & 1642.9 & 1646.1 & 1649.2 & 1652.4 & 1655.6 & 1658.8 & 1662.0 & 1665.2 & 1668.4 & 1671.6 & 4.900 \\
\hline
\end{tabular}

*Based on the International Temperature Scale of 1948. 
TABLE 3-A. Sixty percent iridium-forty percent rhodium versus iridium thermocouples - Continued

Electromotive force in absolute millivolts. Temperatures in degrees $\mathrm{F}^{*}$. Reference junctions at $32^{\circ} \mathrm{F}$.

\begin{tabular}{|c|c|c|c|c|c|c|c|c|c|c|c|}
\hline $\begin{array}{l}\text { Milli- } \\
\text { volts }\end{array}$ & .000 & .010 & .020 & .030 & .040 & .050 & .060 & .070 & .080 & .090 & 100 \\
\hline
\end{tabular}

\begin{tabular}{|c|c|c|c|c|c|c|c|c|c|c|c|c|}
\hline 000 & 1671.6 & & 1678.0 & 1681.2 & & 1687.6 & 1690.8 & 1694.0 & 1697.2 & 1700.4 & 1703.6 & 5.000 \\
\hline 100 & 1703.6 & 1706.8 & 1710.1 & 1713.3 & & 1719.7 & 1723.0 & 1726.2 & 1729.4 & 1732.6 & & 5.100 \\
\hline 200 & 1735.9 & 1739.1 & 1742.3 & 1745.6 & 1748.8 & 1752.0 & 1755.3 & 1758.5 & 1761.8 & 1765.0 & 1768.3 & .200 \\
\hline 5.300 & 1768.3 & 1771.5 & 1774.8 & 1778.0 & 1781.3 & 1784.6 & 1787.8 & 1791.1 & 1794.3 & 1797.6 & 1800.9 & .300 \\
\hline 5.400 & 1800.9 & 1804.1 & 1807.4 & 1810.7 & 1814.0 & 1817.2 & 1820.5 & 1823.8 & 1827.0 & 1830.3 & 1833.6 & \\
\hline 500 & 1833.6 & 1836.9 & 1840.2 & 1843.5 & 1846.8 & 1850.1 & 1853.4 & 1856.7 & 1860.0 & 1863.3 & 1866.6 & 500 \\
\hline .600 & 1866.6 & 1869.9 & 1873.2 & 1876.5 & 1879.8 & 1883.1 & 1886.4 & 1889.7 & 1893.0 & 1896.4 & 1899.7 & .600 \\
\hline 5.700 & 1899.7 & 1903.0 & 1906.3 & 1909.6 & 1912.9 & 1916.3 & 1919.6 & 1922.9 & 1926.3 & 1929.6 & 1932.9 & .700 \\
\hline 5.800 & 1932.9 & 1936.3 & 1939.6 & 1943.0 & 1946.3 & 1949.7 & 1953.0 & 1956.3 & 1959.7 & 1963.0 & 1966.4 & .800 \\
\hline 5.900 & 1966.4 & 1969.8 & 1973.1 & 1976.5 & 1979.8 & 1983.2 & 1986.5 & & & & & \\
\hline .000 & & & & & & & & & & & & .000 \\
\hline 6.100 & 2033.8 & 2037.1 & 2040.5 & 2043.9 & 2047.3 & 2050.7 & 2054.1 & 2057.5 & & & 2067.7 & .100 \\
\hline 6.200 & 2067.7 & 2071.1 & 2074.5 & 2077.9 & 2081.3 & 2084.7 & 2088.1 & 2091.6 & & & & .200 \\
\hline 6.300 & 2101.8 & 210 & 210 & 211 & & & & & & & & 300 \\
\hline 400 & 2136.0 & 213 & 2.9 & 2146.3 & 214 & 215 & & & & & & 400 \\
\hline 500 & 2170.4 & 2173.9 & 2177.3 & 2180.7 & 2184.2 & 2187.6 & 2191.1 & 2194.5 & 8.0 & 1.5 & 2204.9 & .500 \\
\hline 6.600 & 2204.9 & 2208.4 & 2211.8 & 2215.3 & & & & & & & & 6.600 \\
\hline 6.700 & 2239.5 & 2243.0 & 2246.5 & 2250.0 & & & & & & & & 6.700 \\
\hline 6.800 & 2274.3 & & & & & & & & & & & 800 \\
\hline 900 & 2309.2 & 2312.7 & 2316.2 & 2319.7 & 2323.2 & 2326.7 & & & & & & 900 \\
\hline 7.000 & & & & & & & & & & & & 7.000 \\
\hline 7.100 & 2379.2 & 2382.8 & 2386.3 & & & & & & & & & 7.100 \\
\hline 7.200 & 2414.4 & & & & & & & & & & & 7.200 \\
\hline 7.300 & 244 & & & & & & & & & & & 300 \\
\hline 7.400 & 2484.9 & 2488.4 & 2491.9 & 2495.4 & 2499.0 & 250 & 06.0 & 9.6 & & 6.6 & & 400 \\
\hline 7.500 & 2520.2 & & 527.2 & & & & & 4.9 & & & & 7.500 \\
\hline 7.600 & 2555.5 & 2559.1 & 2562.6 & 2566.1 & 2569.7 & & & & & & 2590.9 & 7.600 \\
\hline 7.700 & 2590.9 & & & & & & & & & & & 7.700 \\
\hline 7.800 & & & & & & & & & & & & 800 \\
\hline 7.900 & 2661.7 & 2665.2 & 2668.8 & 72.3 & 2675.8 & 2679.4 & 2.9 & 2686.5 & 2690.0 & 2693.5 & 2697.1 & 7.900 \\
\hline 8.000 & & & & & & & & & & & & .000 \\
\hline 8.100 & & & & & & & & & & & & 3.100 \\
\hline 8.200 & & & & & & & & & & & & 200 \\
\hline 8.300 & 280 & & 0.1 & & & 2820.6 & & 2827.7 & & & 8.2 & .300 \\
\hline 400 & 2838.2 & & & & & & & & & & & .400 \\
\hline 8.500 & 2873.4 & 2876.9 & 2880.4 & 2883.9 & 2887.4 & 2890.9 & 94.4 & 2897.9 & & & 8.4 & 8.500 \\
\hline 8.600 & & & & & & & & & & & & 8.600 \\
\hline 8.700 & & & & & & & & & & & & 700 \\
\hline & & & & & & & & & & & & 8.800 \\
\hline 8.900 & 3013.1 & 3016.6 & 3020.0 & 3023.5 & 3027.0 & 3030.5 & 3033.9 & 3037.4 & 3040.8 & 3044.3 & 3047.8 & 8.900 \\
\hline 9.000 & & & & & & & & & & & & .000 \\
\hline 9.100 & & & & & & & & & & & & .100 \\
\hline 9.200 & & & & & & & & & & & & 9.200 \\
\hline & & & & & & & & & & & & 9.300 \\
\hline 9.400 & & & 3191.9 & & & 320 & & & & & & 9.400 \\
\hline 9.500 & 3219.1 & 3222.5 & 3225.9 & 3229.3 & 3232.7 & 3236.1 & 3239.5 & 3242.8 & 3246.2 & 3249.6 & 3253.0 & 9.500 \\
\hline 9.600 & 3253.0 & 3256.3 & 3259.7 & 3263.1 & 3266.5 & 3269.8 & 3273.2 & 3276.6 & 3279.9 & 3283.3 & 3286.7 & 9.600 \\
\hline 9.700 & 3286.7 & 3290.0 & & & & & & & & & 3320.2 & 9.700 \\
\hline & & & & & & & & & & & & 9.800 \\
\hline 9.900 & 3353.6 & 3356.9 & 3360.3 & 3363.6 & 3366.9 & 3370.2 & 3373.5 & 3376.9 & 3380.2 & 3383.5 & 3386.8 & 9.900 \\
\hline
\end{tabular}

*Based on the International Temperature Scale of 1948. 
TABLE 3-A. Sixty percent iridium-forty percent rhodium versus iridium thermocouples-Continued Electromotive force in absolute millivolts. Temperatures in degrees $\mathrm{F}^{*}$. Reference junctions at $32^{\circ} \mathrm{F}$.

\begin{tabular}{|c|c|c|c|c|c|c|c|c|c|c|c|c|}
\hline \multirow{2}{*}{$\begin{array}{l}\text { Milli- } \\
\text { volts }\end{array}$} & .000 & .010 & .020 & .030 & .040 & .050 & .060 & .070 & .080 & .090 & .100 & \\
\hline & \multicolumn{12}{|c|}{ Degrees F } \\
\hline 10.000 & 3386.8 & 3390.1 & 3393.5 & 3396.8 & 3400.1 & 3403.4 & 3406.7 & 3410.0 & 3413.3 & 3416.6 & 3419.9 & 10.000 \\
\hline 10.100 & 3419.9 & 3423.2 & 3426.5 & 3429.8 & 3433.1 & 3436.4 & 3439.6 & 3442.9 & 3446.2 & 3449.5 & 3452.8 & 10.100 \\
\hline 10.200 & 3452.8 & 3456.1 & 3459.4 & 3462.6 & 3465.9 & 3469.2 & 3472.5 & 3475.7 & 3479.0 & 3482.3 & 3485.6 & 10.200 \\
\hline 10.300 & 3485.6 & 3488.8 & 3492.1 & 3495.4 & 3498.6 & 3501.9 & 3505.1 & 3508.4 & 3511.7 & 3514.9 & 3518.2 & 10.300 \\
\hline 10.400 & 3518.2 & 3521.4 & 3524.7 & 3527.9 & 3531.2 & 3534.4 & 3537.6 & 3540.9 & 3544.1 & 3547.3 & 3550.6 & 10.400 \\
\hline 10.500 & 3550.6 & 3553.8 & 3557.0 & 3560.3 & 3563.5 & 3566.7 & 3570.0 & 3573.2 & 3576.4 & 3579.7 & 3582.9 & 10.500 \\
\hline 10.600 & 3582.9 & 3586.1 & 3589.3 & 3592.5 & 3595.8 & 3599.0 & 3602.2 & 3605.4 & 3608.6 & 3611.8 & 3615.0 & 10.600 \\
\hline 10.700 & 3615.0 & 3618.2 & 3621.4 & 3624.6 & 3627.8 & 3631.0 & 3634.2 & 3637.4 & 3640.6 & 3643.8 & 3647.0 & 10.700 \\
\hline 10.800 & 3647.0 & 3650.2 & 3653.4 & 3656.6 & 3659.7 & 3662.9 & 3666.1 & 3669.3 & 3672.5 & 3675.7 & 3678.9 & 10.800 \\
\hline 10.900 & 3678.9 & 3682.0 & 3685.2 & 3688.4 & 3691.6 & 3694.7 & 3697.9 & 3701.1 & 3704.2 & 3707.4 & 3710.5 & 10.900 \\
\hline 11.000 & 3710.5 & 3713.7 & 3716.9 & 3720.0 & 3723.2 & 3726.3 & 3729.5 & 3732.7 & 3735.8 & 3739.0 & 3742.1 & 11.000 \\
\hline 11.100 & 3742.1 & 3745.3 & 3748.4 & 3751.6 & 3754.7 & 3757.9 & 3761.0 & 3764.2 & 3767.3 & 3770.4 & 3773.6 & 11.100 \\
\hline 11.200 & 3773.6 & 3776.7 & 3779.8 & 3783.0 & 3786.1 & 3789.2 & 3792.4 & 3795.5 & 3798.6 & 3801.8 & 3804.9 & 11.200 \\
\hline 11.300 & 3804.9 & 3808.0 & 3811.1 & 3814.3 & 3817.4 & 3820.5 & 3823.6 & 3826.7 & 3829.8 & 3833.0 & 3836.1 & 11.300 \\
\hline 11.400 & 3836.1 & 3839.2 & 3842.3 & 3845.4 & 3848.5 & 3851.6 & 3854.8 & 3857.9 & 3861.0 & 3864.1 & 3867.2 & 11.400 \\
\hline 11.500 & 3867.2 & 3870.3 & 3873.4 & 3876.5 & 3879.6 & 3882.7 & 3885.8 & 3888.9 & 3892.0 & 3895.0 & 3898.1 & 11.500 \\
\hline 11.600 & 3898.1 & 3901.2 & & & & & & & & & & 11.600 \\
\hline
\end{tabular}

*Based on the International Temperature Scale of 1948. 
TABLE 4-A. Sixty percent iridium-forty percent rhodium versus iridium thermocouples

Electromotive force in absolute millivolts. Temperatures in degrees $\mathrm{F}^{*}$. Reference junctions at $32^{\circ} \mathrm{F}$.

\begin{tabular}{|c|c|c|c|c|c|c|c|c|c|c|c|c|}
\hline \multirow{2}{*}{${ }^{\circ} \mathrm{F}$} & 0 & 10 & 20 & 30 & 40 & 50 & 60 & 70 & 80 & 90 & 100 & \multirow{2}{*}{$F$} \\
\hline & \multicolumn{11}{|c|}{ Millivolts } & \\
\hline 0 & & & & & 0.014 & 0.032 & 0.050 & 0.068 & 0.087 & 0.106 & 0.126 & 0 \\
\hline 100 & 0.126 & 0.146 & 0.167 & 0.187 & 0.209 & 0.230 & 0.252 & 0.274 & 0.297 & 0.319 & 0.342 & 100 \\
\hline 200 & 0.342 & 0.366 & 0.390 & 0.414 & 0.438 & 0.463 & 0.488 & 0.513 & 0.538 & 0.564 & 0.590 & 200 \\
\hline 300 & 0.590 & 0.616 & 0.643 & 0.670 & 0.697 & 0.724 & 0.751 & 0.779 & 0.807 & 0.835 & 0.864 & 300 \\
\hline 400 & 0.864 & 0.892 & 0.921 & 0.950 & 0.979 & 1.008 & 1.038 & 1.067 & 1.097 & 1.127 & 1.158 & 400 \\
\hline 500 & 1.158 & 1.188 & 1.218 & 1.249 & 1.280 & 1.311 & 1.342 & 1.373 & 1.404 & 1.436 & 1.468 & 500 \\
\hline 600 & 1.468 & 1.499 & 1.531 & 1.563 & 1.595 & 1.627 & 1.659 & 1.692 & 1.724 & 1.757 & 1.789 & 600 \\
\hline 700 & 1.789 & 1.822 & 1.855 & 1.888 & 1.921 & 1.954 & 1.987 & 2.020 & 2.053 & 2.086 & 2.119 & 700 \\
\hline 800 & 2.119 & 2.153 & 2.186 & 2.220 & 2.253 & 2.287 & 2.320 & 2.354 & 2.387 & 2.421 & 2.455 & 800 \\
\hline 900 & 2.455 & 2.488 & 2.522 & 2.556 & 2.589 & 2.623 & 2.657 & 2.691 & 2.725 & 2.758 & 2.792 & 900 \\
\hline 1000 & 2.792 & 2.826 & 2.860 & 2.894 & 2.928 & 2.962 & 2.996 & 3.030 & 3.064 & 3.098 & 3.132 & 1000 \\
\hline 1100 & 3.132 & 3.166 & 3.200 & 3.233 & 3.267 & 3.301 & 3.335 & 3.368 & 3.402 & 3.435 & 3.469 & 1100 \\
\hline 1200 & 3.469 & 3.502 & 3.536 & 3.569 & 3.603 & 3.6 & 3.669 & 3.7 & 3.736 & 3.769 & 3.8 & 1200 \\
\hline 1300 & 3.802 & 3.835 & 3.869 & 3.902 & 3.935 & 3.967 & 4.000 & 4.0 & 4.066 & 4.099 & 4.131 & 1300 \\
\hline 1400 & 4.131 & 4.164 & 4.197 & 4.229 & 4.262 & 4.294 & 4.327 & 4.359 & 4.391 & 4.423 & 4.456 & 1400 \\
\hline 1500 & 4.456 & 4.488 & 4.520 & 4.552 & 4.584 & 4.616 & 4.648 & 4.680 & 4.711 & 4.743 & 4.775 & 1500 \\
\hline 1600 & 4.775 & 4.806 & 4.838 & 4.869 & 4.901 & 4.932 & 4.964 & 4.995 & 5.026 & 5.057 & 5.089 & 1600 \\
\hline 1700 & 5.089 & 5.120 & 5.151 & 5.182 & 5.213 & 5.244 & 5.274 & 5.3 & 5.3 & 5.367 & 5.397 & 1700 \\
\hline 1800 & 5.397 & 5.428 & 5.458 & 5.489 & 5.519 & 5.550 & 5.580 & 5.610 & 5.641 & 5.671 & 5.701 & 1800 \\
\hline 1900 & 5.701 & 5.731 & 5.761 & 5.791 & 5.821 & 5.851 & 5.881 & 5.911 & 5.941 & 5.970 & 6.000 & 1900 \\
\hline 2000 & 6.000 & 6.030 & 6.059 & 6.089 & 6.118 & 6.148 & 6.177 & 6.2 & 6.2 & 6.26 & 6.2 & 2000 \\
\hline 2100 & 6.295 & 6.324 & 6.353 & 6.382 & 6.412 & 6.441 & 6.470 & 6.499 & 6.528 & 6.557 & 6.586 & 2100 \\
\hline 2200 & 6.586 & 6.615 & 6.644 & 6.672 & 6.701 & 6.730 & 6.759 & 6.788 & 6.816 & 6.845 & 6.8 & 2200 \\
\hline 2300 & 6.874 & 6.902 & 6.931 & 6.959 & 6.988 & 7.017 & 7.045 & 7.074 & 7.102 & 7.131 & 7.159 & 2300 \\
\hline 2400 & 7.159 & 7.188 & 7.216 & 7.244 & 7.273 & 7.301 & 7.330 & 7.358 & 7.386 & 7.415 & 7.443 & 2400 \\
\hline 2500 & 7.443 & 7.471 & 7.499 & 7.528 & 7.556 & 7.584 & 7.613 & 7.641 & 7.669 & 7.697 & 7.726 & 2500 \\
\hline 2600 & 7.726 & 7.754 & 7.782 & 7.810 & 7.839 & 7.867 & 7.895 & 7.923 & 7.952 & 7.980 & 8.008 & 2600 \\
\hline 2700 & 8.008 & 8.037 & 8.065 & 8.093 & 8.121 & 8.150 & 8.178 & 8.206 & 8.2 & 8.263 & 8.291 & 2700 \\
\hline 2800 & 8.291 & 8.320 & 8.348 & 8.377 & 8.405 & 8.433 & 8.462 & 8.490 & 8.519 & 8.547 & 8.576 & 2800 \\
\hline 2900 & 8.576 & 8.604 & 8.633 & 8.662 & 8.690 & 8.719 & 8.747 & 8.776 & 8.805 & 8.834 & 8.862 & 2900 \\
\hline 3000 & 8.862 & 8.891 & 8.920 & 8.949 & 8.978 & 9.006 & 9.035 & & & & & 3000 \\
\hline 3100 & 9.151 & 9.180 & 9.210 & 9.239 & 9.268 & 9.297 & 9.326 & 9.356 & 9.385 & 9.414 & 9.444 & 3100 \\
\hline 3200 & 9.444 & 9.473 & 9.503 & 9.532 & 9.562 & 9.591 & 9.621 & 9.650 & 9.680 & 9.710 & 9.740 & 3200 \\
\hline 3300 & 9.740 & 9.769 & 9.799 & 9.829 & 9.859 & 9.889 & 9.919 & 9.949 & 9.979 & 10.010 & 10.040 & 3300 \\
\hline 3400 & 10.040 & 10.070 & 10.100 & 10.131 & 10.161 & 10.191 & 10.222 & 10.252 & 10.283 & 10.3 & 10.344 & 3400 \\
\hline 3500 & 10.344 & 10.375 & 10.406 & 10.436 & 10.467 & 10.498 & 10.529 & 10.560 & 10.591 & 10.622 & 10.653 & 3500 \\
\hline 3600 & 10.653 & 10.684 & 10.715 & 10.747 & 10.778 & 10.809 & 10.841 & 10.872 & 10.904 & 10.935 & 10.967 & 3600 \\
\hline 3700 & 10.967 & 10.998 & 11.030 & 11.061 & 11.093 & 11.125 & 11.157 & 11.189 & 11.220 & 11.252 & 11.284 & 3700 \\
\hline 3800 & 11.284 & 11.316 & 11.348 & 11.380 & 11.413 & 11.445 & 11.477 & 11.509 & 11.541 & 11.574 & 11.606 & 3800 \\
\hline 3900 & 11.606 & & & & & & & & & & & 3900 \\
\hline
\end{tabular}

*Based on the International Temperature Scale of 1948. 
TABLE 5-A. Fifty percent iridium-fifty percent rhodium versus iridium thermocouples

Electromotive force in absolute millivolts. Temperatures in degrees C (Int. 1948). Reference junctions at $0{ }^{\circ} \mathrm{C}$.

\begin{tabular}{|c|c|c|c|c|c|c|c|c|c|c|c|c|}
\hline \multirow{2}{*}{$\begin{array}{l}\text { Milli- } \\
\text { volts }\end{array}$} & .000 & .010 & .020 & .030 & .040 & .050 & .060 & .070 & .080 & .090 & .100 & \multirow{2}{*}{$\begin{array}{l}\text { Milli- } \\
\text { volts }\end{array}$} \\
\hline & \multicolumn{11}{|c|}{ Degrees C } & \\
\hline 0.000 & 0.0 & 3.1 & 6.2 & 9.2 & 12.2 & 15.1 & 18.1 & 21.0 & 23.8 & 26.7 & 29.5 & 0.000 \\
\hline 0.100 & 29.5 & 32.2 & 35.0 & 37.7 & 40.4 & & 45.8 & 48.4 & 51.0 & 53.6 & 56.2 & 0.100 \\
\hline 0.200 & 56.2 & 58.7 & 61.3 & 63.8 & 66.3 & 68.8 & 71.2 & 73.7 & 76.1 & 78.5 & 80.9 & 0.200 \\
\hline 0.300 & 80.9 & 83.3 & 85.7 & 88.1 & 90.4 & 92.8 & 95.1 & 97.4 & 99.7 & 102.0 & 104.2 & 0.300 \\
\hline 0.400 & 104.2 & 106.5 & 108.8 & 111.0 & 113.2 & 115.4 & 117.6 & 119.8 & 122.0 & 124.2 & 126.4 & 0.400 \\
\hline 0.500 & 126.4 & 128.5 & 130.7 & 132.8 & 135.0 & 137.1 & 139.2 & 141.3 & 143.4 & 145.5 & 147.6 & 0.500 \\
\hline 0.600 & 147.6 & 149.7 & 151.8 & 153.8 & 155.9 & 157.9 & 160.0 & 162.0 & 164.0 & 166.1 & 168.1 & 0.600 \\
\hline 0.700 & 168.1 & 170.1 & 172.1 & 174.1 & 176.1 & 178.1 & 180.0 & 182.0 & 184.0 & 185.9 & 187.9 & 0.700 \\
\hline 0.800 & 187.9 & 189.8 & 191.8 & 193.7 & 195.7 & 197.6 & 199.5 & 201.5 & 203.4 & 205.3 & 207.2 & 0.800 \\
\hline 0.900 & 207.2 & 209.1 & 211.0 & 212.9 & 214.8 & 216.7 & 218.5 & 220.4 & 222.3 & 224.2 & 226.0 & 0.900 \\
\hline .000 & 226.0 & 227.9 & 229.7 & 231.6 & 233.4 & & 237.1 & 239.0 & 240.8 & 242.6 & 244.4 & 1.000 \\
\hline 1.100 & 244.4 & 246.3 & 248.1 & 249.9 & 251.7 & 253.5 & 255.3 & 257.1 & 258. & 26 & & 1.100 \\
\hline 1.200 & 262.5 & 264.3 & 266.1 & 267.9 & 269.7 & 271.5 & 273.3 & 275.0 & 276.8 & 278.6 & 280.3 & 1.200 \\
\hline 1.300 & 280.3 & 282.1 & 283.9 & 285.6 & 287.4 & 289.1 & 290.9 & 292.6 & 294.4 & 296.1 & 297.9 & 1.300 \\
\hline 1.400 & 297.9 & 299.6 & 301.3 & 303.1 & 304.8 & 306.5 & 308.3 & 310.0 & 311.7 & 313.4 & 315.2 & 1.400 \\
\hline 1.500 & 315.2 & 316.9 & 318.6 & 320.3 & 322.0 & 323.7 & 325.5 & 327 & 328.9 & 330.6 & 332.3 & 1.500 \\
\hline 1.600 & 332.3 & 334.0 & 335.7 & 337.4 & 339.1 & 340 & 342.5 & 344 & 345 & 34 & .2 & 1.600 \\
\hline 1.700 & 349.2 & 350.9 & 352.6 & 354.3 & 355.9 & 357.6 & 359.3 & 361.0 & 362.6 & 364.3 & 366.0 & 1.700 \\
\hline 1.800 & 366.0 & 367.7 & 369.3 & 371.0 & 372.7 & 374.3 & 376.0 & 377.6 & 379.3 & 381.0 & 382.6 & 1.800 \\
\hline 1.900 & 382.6 & 384.3 & 385.9 & 387.6 & 389.2 & 390.9 & 392.6 & 394.2 & 395.9 & 397.5 & 399.2 & 1.900 \\
\hline 000 & 9.2 & 00.8 & 402.4 & 04. & & & & & & & & 2.000 \\
\hline .100 & 415.6 & 417.2 & 418.9 & 420.5 & 422.1 & 423.8 & 425.4 & 427.0 & 428.7 & 430.3 & & 2.100 \\
\hline 2.200 & 431.9 & 433.6 & 435.2 & 436.8 & 438.4 & 440.1 & 441.7 & 443.3 & 445.0 & 446.6 & 448.2 & 2.200 \\
\hline 2.300 & 448.2 & 449.8 & 451.5 & 453.1 & 454.7 & 456.3 & 457.9 & 459.6 & 461.2 & 462.8 & 464.4 & 2.300 \\
\hline 2.400 & 464.4 & 466.0 & 467.7 & 469.3 & 470.9 & 472.5 & 474.1 & 475.7 & 477.4 & 479.0 & 480.6 & 2.400 \\
\hline .500 & 480.6 & 482.2 & 483.8 & 485.4 & 487.0 & 488.7 & 490 & 49 & 493 & & & 2.500 \\
\hline 2.600 & 496.7 & 498.3 & 499.9 & 501.5 & 503.2 & 504.8 & 506.4 & 508.0 & 509 & 511.2 & 512.8 & 2.600 \\
\hline 2.700 & 512.8 & 514.4 & 516.0 & 517.6 & 519.2 & 520.9 & 522.5 & 524.1 & 525.7 & 527.3 & 528.9 & 2.700 \\
\hline 2.800 & 528.9 & 530.5 & 532.1 & 533.7 & 535.3 & 536. & 538 & 540 & 54 & 543.4 & & 2.800 \\
\hline 2.900 & 545.0 & 546.6 & 548.2 & 549.8 & 551.4 & 553.0 & 554.6 & 556.2 & 557.8 & 559.4 & 561.0 & 2.900 \\
\hline 000 & 1.0 & 62.6 & 564.2 & 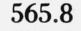 & & & & & & & & 3.000 \\
\hline .100 & 577.1 & 578.7 & 580.3 & 581.9 & 583.5 & 585. & 586.7 & 588.3 & 589.9 & 591.6 & 593.2 & 3.100 \\
\hline 3.200 & 593.2 & 594.8 & 596.4 & 598.0 & 599.6 & 601 & 602 & 60 & 60 & 607.6 & & 3.200 \\
\hline 3.300 & 609.2 & 610.9 & 612.5 & & & & & & & & & 3.300 \\
\hline 3.400 & & & & & & & & & & & & 3.400 \\
\hline 3.500 & 641.5 & 643.1 & 644.7 & 646.3 & 647.9 & & & & & 56.0 & & 3.500 \\
\hline 3.600 & 657.6 & 659.2 & 660.8 & 662.4 & 664.0 & 665.7 & 667.3 & 668.9 & 670.5 & 672.1 & 673.8 & 3.600 \\
\hline 3.700 & 673.8 & 675.4 & 677.0 & 678.6 & 680.2 & 681.9 & 683.5 & 685.1 & 686.7 & 688.4 & 690.0 & 3.700 \\
\hline 3.800 & 690.0 & 691.6 & 693.2 & 694.9 & 696.5 & 698.1 & 699 & 701.4 & 70 & 704.6 & & 3.800 \\
\hline 3.900 & 706.3 & 707.9 & 709.5 & 711.2 & 712.8 & 714.4 & 716.1 & 717.7 & 719.3 & 721.0 & 722.6 & 3.900 \\
\hline 4.000 & 2.6 & 724 & & & 7 & & & & & & & 4.000 \\
\hline 4.100 & 739.0 & 740.6 & 742.3 & 743.9 & 745.6 & 747.2 & 748 & 750.5 & 752 & 753.8 & & 4.100 \\
\hline 4.200 & 755.4 & 757.1 & 758.7 & 760.4 & 762.0 & 763.7 & 765.3 & 767.0 & 768.6 & 770.3 & 771.9 & 4.200 \\
\hline 4.300 & 771.9 & 773.6 & 775.2 & 776.9 & & 780.2 & & & & & 788.5 & 4.300 \\
\hline 4.400 & 788.5 & 790.1 & 791.8 & & & & & & & & & 4.400 \\
\hline 4.500 & 805.1 & 806.8 & 808.5 & 810.1 & 811.8 & 813.5 & & & & & & 4.500 \\
\hline 4.600 & 821.8 & 823.5 & 825.2 & 826.8 & 828.5 & 830.2 & 831.9 & & & 836.9 & & 4.600 \\
\hline 4.700 & 838.6 & 840.3 & 841.9 & 843.6 & 845.3 & 847.0 & 848.7 & 850.4 & 852.1 & 853.7 & 855.4 & 4.700 \\
\hline 4.800 & 855.4 & 857.1 & 858.8 & 860.5 & 862.2 & 863.9 & 865.6 & 867.3 & 869.0 & 870.7 & 872.3 & 4.800 \\
\hline 4.900 & 872.3 & 874.0 & 875.7 & 877.4 & 879.1 & 880.8 & 882.5 & 884.2 & 885.9 & 887.6 & 889.3 & 4.900 \\
\hline
\end{tabular}


TABLE 5-A. Fifty percent iridium-fifty percent rhodium versus iridium thermocouples-Continued

Electromotive force in absolute millivolts. Temperatures in degrees C (Int. 1948). Reference junctions at $0{ }^{\circ} \mathrm{C}$.

\begin{tabular}{|c|c|c|c|c|c|c|c|c|c|c|c|c|}
\hline \multirow{2}{*}{$\begin{array}{l}\text { Milli- } \\
\text { volts }\end{array}$} & .000 & .010 & .020 & .030 & .040 & .050 & .060 & .070 & .080 & .090 & .100 & \multirow{2}{*}{$\begin{array}{l}\text { Milli- } \\
\text { volts }\end{array}$} \\
\hline & \multicolumn{11}{|c|}{ Degrees C } & \\
\hline 000 & 889.3 & 891.0 & 892.7 & 894.5 & 896.2 & 897.9 & 899.6 & 901.3 & 903.0 & 904.7 & 906.4 & 5.000 \\
\hline 100 & 906.4 & 908.1 & 909.8 & 911.5 & 913.3 & 915.0 & 916.7 & 918.4 & 920.1 & 921.8 & 923.6 & 5.100 \\
\hline 200 & 923.6 & 925.3 & 927.0 & 928.7 & 930.4 & 932.2 & 933.9 & 935.6 & 937.3 & 939.0 & 940.8 & 5.200 \\
\hline .300 & 940.8 & 942.5 & 944.2 & 946.0 & 947.7 & 949.4 & 951.1 & 952.9 & 954.6 & 956.3 & 958.1 & 5.300 \\
\hline 400 & 958.1 & 959.8 & 961.5 & 963.3 & 965.0 & 966.8 & 968.5 & 970.2 & 972.0 & & & 5.400 \\
\hline 500 & 975.5 & 977.2 & 978.9 & 980.7 & 982.4 & 984.2 & 985.9 & 987.7 & 989.4 & 991.2 & 992.9 & 5.500 \\
\hline 600 & 992.9 & 994.7 & 996.4 & 998.2 & 999.9 & 1001.7 & 1003.4 & 1005 & 1006.9 & 1008.7 & 1010.4 & 5.600 \\
\hline .700 & 1010.4 & 1012.2 & 1014.0 & 1015.7 & 1017.5 & 1019.2 & 21.0 & .8 & 4.5 & 5.3 & .1 & .700 \\
\hline 5.800 & 1028.1 & 1029.8 & 1031.6 & 1033.4 & 1035.1 & 1036.9 & 1038.7 & 1040.4 & 1042.2 & 1044.0 & 5.7 & 5.800 \\
\hline 5.900 & 1045.7 & 1047.5 & 1049.3 & 1051.1 & 1052.8 & 1054.6 & 1056.4 & 1058.2 & 1059.9 & 1061.7 & 1063.5 & 5.900 \\
\hline 6.000 & 10635 & & & & & & & & & & & 6.000 \\
\hline 100 & 81.3 & 1083 & 9 & 7 & & & & & & & & 6.100 \\
\hline 200 & 1099.2 & 1101.0 & 02.8 & 1104.6 & 1106 & 110 & 0 & 8 & & & & .200 \\
\hline 300 & 1117.2 & 1119.0 & 20.8 & 1122.6 & 112 & 112 & & & & & & 300 \\
\hline 6.400 & 1135.2 & 1137.0 & 1138.8 & 140.6 & 1142.4 & 1144.2 & & & & & & 6.400 \\
\hline 6.500 & 1153.3 & 1155.1 & 1156.9 & 1158.7 & 1160.5 & 1162.3 & 1164.1 & 1166.0 & 1167.8 & & 1171.4 & 6.500 \\
\hline 600 & 1171.4 & 1173.2 & 1175.0 & 1176.8 & 1178.7 & & & & & & 1189.6 & 6.600 \\
\hline 700 & 189.6 & .4 & & 1 & & & & & & & & .700 \\
\hline 800 & 1207.8 & 1209.6 & 11.5 & 1213.3 & 1215.1 & 1216.9 & 8 & 122 & 2.4 & & & .800 \\
\hline 6.900 & 1226.1 & 1227.9 & 1229.7 & 1231.6 & 1233.4 & 1235.2 & 1237.1 & 1238.9 & 1240.7 & 124 & & 6.900 \\
\hline 7.000 & & & & & & & & & & & & 7.000 \\
\hline 7.100 & & & & & & & & & & & & 7.100 \\
\hline 7.200 & 1281.1 & 32.9 & 34.8 & 6 & & & & & & & & 7.200 \\
\hline 7.300 & 1299.5 & 1301.3 & 03.1 & 305.0 & 06.8 & 1308.7 & 10.5 & 3 & 2 & & & 7.300 \\
\hline 7.400 & 1317.9 & 1319.7 & 21.5 & 23.4 & & 1327.1 & & & & & & 7.400 \\
\hline 7.500 & 1336.3 & 1338.1 & 340.0 & 1341.8 & 1343.6 & 1345.5 & 1347.3 & 1349.2 & .0 & 1352.8 & 1.7 & 7.500 \\
\hline 7.600 & 1354.7 & 1356.5 & 358.4 & 1360.2 & 1362.0 & & & & & 1371.3 & 1373.1 & 7.600 \\
\hline 7.700 & 1373.1 & & & & & & & & & & & 7.700 \\
\hline 7.800 & 1391.5 & 1393.3 & 1395.2 & 1397.0 & & 1400.7 & & & & & & 7.800 \\
\hline 7.900 & 1409.9 & 1411.7 & 1413.6 & 1415.4 & 1417.2 & 1419.1 & 1420.9 & 1422.7 & 1424.6 & 1426.4 & 1428.2 & 7.900 \\
\hline 8.000 & & & & & & & & & & & & 8.000 \\
\hline 8.100 & & & & & & & & & & & & 8.100 \\
\hline 200 & & & & & & & & & & & & .200 \\
\hline 300 & 83.2 & 85.0 & 36.8 & 38.7 & 1 & 1492.3 & & & & & & 3.300 \\
\hline 400 & 1501.4 & 1503.2 & 1505.1 & 6.9 & & & & & 6.0 & 8 & & 3.400 \\
\hline 8.500 & 1519.6 & 1521.4 & 1523.2 & 1525.1 & 1526.9 & 1528.7 & 1530.5 & 1532.3 & 1534.1 & 1535.9 & & 8.500 \\
\hline 8.600 & 1537.8 & 1539.6 & 1541.4 & 1543.2 & 1545.0 & & & & 1552.2 & & & 8.600 \\
\hline 8.700 & & & & & & & & & & & & 8.700 \\
\hline & & & & & & & & & & & & 8.800 \\
\hline 8.900 & 1591.9 & 1593.6 & 1595.4 & 1597.2 & 1599.0 & 1600.8 & 1602.6 & 1604.4 & 1606.2 & 1608.0 & 1609.8 & 8.900 \\
\hline 9.000 & & & & & & & & & & & & 9.000 \\
\hline 9.100 & 1627.6 & 1629.4 & 1631.2 & 1632.9 & 1634.7 & 1636.5 & 1638.3 & & & & & 9.100 \\
\hline 9.200 & 1645.4 & & & & & & & & & & & 9.200 \\
\hline 9.300 & 1663.1 & 1664.8 & & & & & & & & & & 9.300 \\
\hline & 1680.7 & & & & & & & & & & & 9.400 \\
\hline 9.500 & 1698.2 & 1700.0 & 1701.7 & 1703.5 & 1705.2 & 1707.0 & & & & & & 9.500 \\
\hline 9.600 & 1715.7 & 1717.4 & 1719.2 & 1720.9 & 1722.7 & 1724.4 & 1726.1 & 1727.9 & 1729.6 & 1731.4 & 1733.1 & 9.600 \\
\hline 9.700 & 1733.1 & 1734.8 & 1736.6 & 1738.3 & 1740.0 & 1741.8 & 1743.5 & 1745.2 & 1747.0 & 1748.7 & 1750.4 & 9.700 \\
\hline 9.800 & 1750.4 & 1752.1 & 1753.9 & & & 1759.0 & 1760.8 & 1762.5 & 1764.2 & 1765.9 & & 9.800 \\
\hline 9.900 & 1767.6 & 1769.4 & 1771.1 & 1772.8 & 1774.5 & 1776.2 & 1778.0 & 1779.7 & 1781.4 & 1783.1 & 1784.8 & 9.900 \\
\hline
\end{tabular}


TABLE 5-A. Fifty percent iridium-fifty percent rhodium versus iridium thermocouples-Continued

Electromotive force in absolute millivolts. Temperatures in degrees C (Int. 1948). Reference junctions at $0{ }^{\circ} \mathrm{C}$.

\begin{tabular}{|c|c|c|c|c|c|c|c|c|c|c|c|c|}
\hline \multirow{2}{*}{$\begin{array}{l}\text { Milli- } \\
\text { volts }\end{array}$} & .000 & 010 & .020 & .030 & .040 & .050 & .060 & .070 & .080 & .090 & . 100 & \multirow{2}{*}{$\begin{array}{l}\text { Milli- } \\
\text { volts }\end{array}$} \\
\hline & \multicolumn{11}{|c|}{ Degrees C } & \\
\hline 000 & 1784.8 & 1786.5 & 1788.2 & 1789.9 & 1791.6 & 1793.4 & 1795.1 & 1796.8 & 1798.5 & 1800 & 1801.9 & 10.000 \\
\hline 100 & 1801.9 & 1803.6 & 1805.3 & 1807.0 & 1808.7 & 1810.4 & 1812.1 & 181 & 1815.5 & .2 & 1818.9 & 10.100 \\
\hline 200 & 1818.9 & 1820.6 & 1822.3 & 1824.0 & 1825.7 & 1827.4 & 1829.1 & 1830.7 & 1832.4 & 1834.1 & 183 & 10.200 \\
\hline 300 & 1835.8 & 1837.5 & 1839.2 & 1840.9 & 1842.6 & 1844.3 & 1845.9 & 1847.6 & 1849.3 & 1851.0 & 1852.7 & 10.300 \\
\hline 400 & 1852.7 & 1854.4 & 1856.0 & 1857.7 & 1859.4 & 1861.1 & & & & & & 10.400 \\
\hline 500 & 1869.4 & 1871.1 & 1872.8 & 1874 & 187 & & & & & & & \\
\hline .600 & 1886.2 & 1887.8 & 1889.5 & 1891.2 & 1892.8 & 1894.5 & 1896.1 & 189 & 189 & & & 10. \\
\hline .700 & 1902.8 & 1904.5 & 1906.1 & 1907.8 & 1909.4 & 1911.1 & 1912.7 & 1914.4 & 1916.1 & 1917.7 & 1919.4 & 10.700 \\
\hline 0.800 & 1919.4 & 1921.0 & 1922.7 & 1924.3 & 1926.0 & 1927.6 & & 1931 & & & & 10. \\
\hline 0.900 & 1935.9 & 1937.5 & 1939.2 & 1940.8 & 1942.4 & 1944.1 & 1945.7 & 1947.4 & 1949.0 & 1950.7 & 1952.3 & 10.900 \\
\hline .000 & & & & & & & & & & & & \\
\hline . 100 & 1968.7 & 1970.3 & 1972.0 & 1973.6 & 1975.2 & 1976.9 & 1978.5 & 1980.1 & 1981.8 & 198 & & 11.100 \\
\hline .200 & 1985.0 & 1986.6 & 1988.3 & 1989.9 & 199 & 199 & & & & & & \\
\hline 1.300 & 2001.3 & 2002.9 & 2004.5 & 2006.2 & 2007.8 & 200 & & 2012.7 & & & & 11.300 \\
\hline 1.400 & 2017.5 & 2019.1 & 2020.8 & 2022.4 & & & & & & & & 11.400 \\
\hline 1.500 & 2033.7 & & 2036.9 & 2038 & 204 & & & & & & & \\
\hline 1.600 & 2049.8 & 2051.4 & 2053.1 & 2054.7 & 2056.3 & 2057.9 & 2059.5 & 2061.1 & 2062.7 & 2064.3 & 5.9 & 11.600 \\
\hline 1.700 & 2065.9 & 2067.6 & 2069.2 & 2070.8 & 2072.4 & 2074.0 & & 2077.2 & & & & 11. \\
\hline 1.800 & 2082.0 & 2083.6 & 2085.2 & 2086.8 & 2088.4 & 2090.0 & 2091.6 & 2093.2 & 2094.9 & 2096.5 & 2098.1 & 11.800 \\
\hline 1.900 & 2098.1 & 2099.7 & 2101.3 & 2102.9 & 2104.5 & 2106.1 & 2107.7 & 2109.3 & 2110.9 & 2112.5 & 2114.1 & 11.900 \\
\hline 2.000 & & & & & & & & & & & & \\
\hline & & & 2133.3 & 2134.9 & 2136.5 & 2138.1 & 2139.7 & 2141.3 & 2142.9 & 2144.5 & 2146.1 & \\
\hline 2.200 & 2146.1 & 2147.7 & 2149.3 & & & & & & & & & 12.200 \\
\hline
\end{tabular}

TABLE 6-A. Fifty percent iridium-fifty percent rhodium versus iridium thermocouples

Electromotive force in absolute millivolts. Temperatures in degrees $\mathrm{C}$ (Int. 1948). Reference junctions at $0{ }^{\circ} \mathrm{C}$.

\begin{tabular}{|c|c|c|c|c|c|c|c|c|c|c|c|c|}
\hline \multirow{2}{*}{${ }^{\circ} \mathrm{C}$} & 0 & 10 & 20 & 30 & 40 & 50 & 60 & 70 & 80 & 90 & 100 & \multirow{2}{*}{${ }^{\circ} \mathrm{C}$} \\
\hline & \multicolumn{11}{|c|}{ Millivolts } & \\
\hline 0 & 0.000 & 0.033 & 0.067 & 0.102 & 0.138 & 0.176 & 0.215 & 0.255 & 0.296 & 0.338 & 0.381 & 0 \\
\hline 100 & 0.381 & 0.426 & 0.471 & 0.517 & 0.564 & 0.612 & 0.660 & 0.710 & 0.760 & 0.811 & 0.862 & 100 \\
\hline 200 & 0.862 & 0.915 & 0.968 & 1.021 & 1.076 & 1.130 & 1.186 & 1.242 & 1.298 & 1.355 & 1.412 & 200 \\
\hline 300 & 1.412 & 1.470 & 1.528 & 1.587 & 1.646 & 1.705 & 1.764 & 1.824 & 1.884 & 1.945 & 2.005 & 300 \\
\hline 400 & 2.005 & 2.066 & 2.127 & 2.188 & 2.250 & 2.311 & 2.373 & 2.434 & 2.496 & 2.558 & 2.620 & 400 \\
\hline 500 & 2.620 & 2.683 & 2.745 & 2.807 & 2.869 & 2.931 & 2.994 & 3.056 & 3.118 & 3.180 & 3.243 & 500 \\
\hline 600 & 3.243 & 3.305 & 3.367 & 3.429 & 3.491 & 3.553 & 3.615 & 3.677 & 3.738 & 3.800 & 3.862 & 600 \\
\hline 700 & 3.862 & 3.923 & 3.984 & 4.045 & 4.106 & 4.167 & 4.228 & 4.288 & 4.349 & 4.409 & 4.469 & 700 \\
\hline 800 & 4.469 & 4.529 & 4.589 & 4.649 & 4.708 & 4.768 & 4.827 & 4.886 & 4.945 & 5.004 & 5.063 & 800 \\
\hline 900 & 5.063 & 5.121 & 5.179 & 5.237 & 5.296 & 5.353 & 5.411 & 5.469 & 5.526 & 5.583 & 5.640 & 900 \\
\hline 1000 & 5.640 & 5.697 & 5.754 & 5.811 & 5.868 & 5.924 & 5.980 & 6.037 & 6.093 & 6.149 & 6.204 & 1000 \\
\hline 1100 & 6.204 & 6.260 & 6.316 & 6.371 & 6.427 & 6.482 & 6.537 & 6.592 & 6.647 & 6.702 & 6.757 & 1100 \\
\hline 1200 & 6.757 & 6.812 & 6.867 & 6.921 & 6.976 & 7.031 & 7.085 & 7.140 & 7.194 & 7.249 & 7.303 & 1200 \\
\hline 1300 & 7.303 & 7.357 & 7.412 & 7.466 & 7.520 & 7.575 & 7.629 & 7.683 & 7.738 & 7.792 & 7.846 & 1300 \\
\hline 1400 & 7.846 & 7.901 & 7.955 & 8.010 & 8.064 & 8.119 & 8.173 & 8.228 & 8.283 & 8.337 & 8.392 & 1400 \\
\hline 1500 & 8.392 & 8.447 & 8.502 & 8.557 & 8.612 & 8.668 & 8.723 & 8.778 & 8.834 & 8.890 & 8.945 & 1500 \\
\hline 1600 & 8.945 & 9.001 & 9.057 & 9.114 & 9.170 & 9.226 & 9.283 & 9.339 & 9.396 & 9.453 & 9.510 & 1600 \\
\hline 1700 & 9.510 & 9.567 & 9.625 & 9.682 & 9.740 & 9.798 & 9.856 & 9.914 & 9.972 & 10.030 & 10.089 & 1700 \\
\hline 1800 & 10.089 & 10.148 & 10.207 & 10.266 & 10.325 & 10.384 & 10.444 & 10.503 & 10.563 & 10.623 & 10.683 & 1800 \\
\hline 1900 & 10.683 & 10.743 & 10.804 & 10.864 & 10.925 & 10.986 & 11.047 & 11.108 & 11.169 & 11.231 & 11.292 & 1900 \\
\hline 2000 & 11.292 & 11.354 & 11.415 & 11.477 & 11.539 & 11.601 & 11.663 & 11.725 & 11.787 & 11.850 & 11.912 & 2000 \\
\hline 2100 & 11.912 & 11.974 & 12.037 & 12.099 & 12.162 & 12.224 & & & & & & 2100 \\
\hline
\end{tabular}


TABLE 7-A. Fifty percent iridium-fifty percent rhodium versus iridium thermocouples

Electromotive force in absolute millivolts. Temperatures in degrees $\mathrm{F}^{*}$. Reference junctions at $32^{\circ} \mathrm{F}$.

\begin{tabular}{|c|c|c|c|c|c|c|c|c|c|c|c|c|}
\hline \multirow{2}{*}{$\begin{array}{l}\text { Milli- } \\
\text { volts }\end{array}$} & .000 & .010 & .020 & .030 & .040 & .050 & .060 & .070 & .080 & .090 & .100 & \multirow{2}{*}{$\begin{array}{l}\text { Milli- } \\
\text { volts }\end{array}$} \\
\hline & \multicolumn{11}{|c|}{ Degrees F } & \\
\hline 0.000 & 32.0 & 37.6 & 43.1 & 48.6 & 53.9 & 59.3 & 64.5 & 69.7 & 74.9 & 80.0 & 85.0 & 0.000 \\
\hline 0.100 & 85.0 & 90.0 & 95.0 & 99.9 & 104.8 & 109.6 & 114.4 & 119.1 & 123.8 & 128.5 & 133.1 & 0.100 \\
\hline 0.200 & 133.1 & 137.7 & 142.3 & 146.8 & 151.3 & 155.8 & 160.2 & 164.6 & 169.0 & 173.4 & 177.7 & 0.200 \\
\hline 0.300 & 177.7 & 182.0 & 186.3 & 190.5 & 194.8 & 199.0 & 203.1 & 207.3 & 211.4 & 215.5 & 219.6 & 0.300 \\
\hline 0.400 & 219.6 & 223.7 & 227.8 & 231.8 & 235.8 & 239.8 & 243.8 & 247.7 & 251.7 & 255.6 & 259.5 & 0.400 \\
\hline 0.500 & 259.5 & 263.4 & 267.3 & 271.1 & 275.0 & 278.8 & 282.6 & 286.4 & 290.2 & 293.9 & 297.7 & 0.500 \\
\hline 0.600 & 297.7 & 301.4 & 305.2 & 308.9 & 312.6 & 316.3 & 319.9 & 323.6 & 327.3 & 330.9 & 334.5 & 0.600 \\
\hline 0.700 & 334.5 & 338.1 & 341.7 & 345.3 & 348.9 & 352.5 & 356.1 & 359.6 & 363.2 & 366.7 & 370.2 & 0.700 \\
\hline 0.800 & 370.2 & 373.7 & 377.2 & 380.7 & 384.2 & 387.7 & 391.2 & 394.6 & 398.1 & 401.5 & 404.9 & 0.800 \\
\hline 0.900 & 404.9 & 408.4 & 411.8 & 415.2 & 418.6 & 422.0 & 425.4 & 428.7 & 432.1 & 435.5 & 438.8 & 0.900 \\
\hline 1.000 & 438.8 & 442.2 & 445.5 & 448.9 & 452.2 & 455.5 & 458.8 & 462.1 & 465.4 & 468.7 & 472.0 & 1.000 \\
\hline 1.100 & 472.0 & 475.3 & 478.6 & 481.8 & 485.1 & 488.4 & 491.6 & 494.9 & 498.1 & 501.3 & 504.6 & 1.100 \\
\hline 1.200 & 504.6 & 507.8 & 511.0 & 514.2 & 517.4 & 520.6 & 523.8 & 527.0 & 530.2 & 533.4 & 536.6 & 1.200 \\
\hline 1.300 & 536.6 & 539.8 & 542.9 & 546.1 & 549.3 & 552.4 & 555.6 & 558.7 & 561.9 & 565.0 & 568.2 & 1.300 \\
\hline 1.400 & 568.2 & 571.3 & 574.4 & 577.5 & 580.7 & 583.8 & 586.9 & 590.0 & 593.1 & 596.2 & 599.3 & 1.400 \\
\hline 1.500 & 599.3 & 602.4 & 605.5 & 608.6 & 611.7 & 614.7 & 617.8 & 620.9 & 624.0 & 627.0 & 630.1 & 1.500 \\
\hline 1.600 & 630.1 & 633.2 & 636.2 & 639.3 & 642.3 & 645.4 & 648.4 & 651.5 & 654.5 & 657.5 & 660.6 & 1.600 \\
\hline 1.700 & 660.6 & 663.6 & 666.6 & 669.7 & 672.7 & 675.7 & 678.7 & 681.7 & 684.7 & 687.8 & 690.8 & 1.700 \\
\hline 1.800 & 690.8 & 693.8 & 696.8 & 699.8 & 702.8 & 705.8 & 708.8 & 711.8 & 714.7 & 717.7 & 720.7 & 1.800 \\
\hline 1.900 & 720.7 & 723.7 & 726.7 & 729.7 & 732.6 & 735.6 & 738.6 & 741.6 & 744.5 & 747.5 & 750.5 & 1.900 \\
\hline 2.000 & 750.5 & 753.4 & 756.4 & 759.4 & 762.3 & 765.3 & 768.2 & 771.2 & 774.1 & & 780.1 & 2.000 \\
\hline 2.100 & 780.1 & 783.0 & 785.9 & 788.9 & 791.8 & 794.8 & 797.7 & 800.7 & 803.6 & 806.5 & 809.5 & 2.100 \\
\hline 2.200 & 809.5 & 812.4 & 815.3 & 818.3 & 821.2 & 824.1 & 827.1 & 830.0 & 832.9 & 835.8 & 838.8 & 2.200 \\
\hline 2.300 & 838.8 & 841.7 & 844.6 & 847.5 & 850.5 & 853.4 & 856.3 & 859.2 & 862.1 & 865.0 & 868.0 & 2.300 \\
\hline 2.400 & 868.0 & 870.9 & 873.8 & 876.7 & 879.6 & 882.5 & 885.4 & 888.3 & 891.2 & 894.1 & 897.1 & 2.400 \\
\hline 2.500 & 897.1 & 900.0 & 902.9 & 905.8 & 908.7 & 911.6 & 914.5 & 917.4 & 920.3 & 923.2 & 926.1 & 2.500 \\
\hline 2.600 & 926.1 & 929.0 & 931.9 & 934.8 & 937.7 & 940.6 & 943.5 & 946.4 & 949.3 & 952.2 & 955.1 & 2.600 \\
\hline 2.700 & 955.1 & 958.0 & 960.9 & 963.8 & 966.6 & 969.5 & 972.4 & 975.3 & 978.2 & 981.1 & 984.0 & 2.700 \\
\hline 2.800 & 984.0 & 986.9 & 989.8 & 992.7 & 995.6 & 998.5 & 1001.4 & 1004.3 & 1007.1 & 1010.0 & 1012.9 & 2.800 \\
\hline 2.900 & 1012.9 & 1015.8 & 1018.7 & 1021.6 & 1024.5 & 1027.4 & 1030.3 & 1033.2 & 1036.1 & 1038.9 & 1041.8 & 2.900 \\
\hline 3.000 & 1041.8 & 1044.7 & 1047.6 & 1050.5 & 53.4 & 1056 & & 62.1 & 1065.0 & & & .000 \\
\hline 3.100 & 1070.8 & 1073.7 & 1076.6 & 1079.4 & 1082.3 & 1085.2 & 1088.1 & 1091.0 & 1093.9 & 1096.8 & 1099.7 & 3.100 \\
\hline 3.200 & 1099.7 & 1102.6 & 1105.5 & 1108.4 & 1111.2 & 1114.1 & 1117.0 & 1119.9 & 1122.8 & 1125.7 & 1128.6 & 3.200 \\
\hline 3.300 & 1128.6 & 1131.5 & 1134.4 & 1137.3 & 1140.2 & 1143.1 & 114.6 .0 & 1148.9 & 1151.8 & 1154.7 & 1157.6 & 3.300 \\
\hline 3.400 & 1157.6 & 1160.5 & 1163.4 & 1166.3 & 1169.2 & 1172.1 & 1175.0 & 1177.9 & 1180.8 & 1183.7 & 1186.6 & 3.400 \\
\hline 3.500 & 1186.6 & 1189.5 & 1192.4 & 1195.3 & 1198.2 & 1201.1 & 1204.0 & 1206.9 & 1209.8 & 1212.7 & 1215.6 & 3.500 \\
\hline 3.600 & 1215.6 & 1218.5 & 1221.5 & 1224.4 & 1227.3 & 1230.2 & 1233.1 & 1236.0 & 1238.9 & 1241.9 & 1244.8 & 3.600 \\
\hline 3.700 & $1244: 8$ & 1247.7 & 1250.6 & 1253.5 & 1256.4 & 1259.4 & 1262.3 & 1265.2 & 1268.1 & 1271.1 & 1274.0 & 3.700 \\
\hline 3.800 & 1274.0 & 1276.9 & 1279.8 & 1282.8 & 1285.7 & 1288.6 & 1291.6 & 1294.5 & 1297.4 & 1300.4 & 1303.3 & 3.800 \\
\hline 3.900 & 1303.3 & 1306.2 & 1309.2 & 1312.1 & 1315.0 & 1318.0 & 1320.9 & 1323.8 & 1326.8 & 1329.7 & 1332.7 & 3.900 \\
\hline 4.000 & 1332.7 & 1335.6 & 1338.6 & 1341.5 & & 1347.4 & 1350.4 & 1353.3 & 1356.3 & & & 4.000 \\
\hline 4.100 & 1362.2 & 1365.1 & 1368.1 & 1371.0 & 1374.0 & 1377.0 & 1379.9 & 1382.9 & 1385.8 & 1388.8 & 1391.8 & 4.100 \\
\hline 4.200 & 1391.8 & 1394.7 & 1397.7 & 1400.7 & 1403.6 & 1406.6 & 1409.6 & 1412.5 & 1415.5 & 1418.5 & 1421.5 & 4.200 \\
\hline 4.300 & 1421.5 & 1424.4 & 1427.4 & 1430.4 & 1433.4 & 1436.4 & 1439.3 & 1442.3 & 1445.3 & 1448.3 & 1451.3 & 4.300 \\
\hline 4.400 & 1451.3 & 1454.3 & 1457.3 & 1460.2 & 1463.2 & 1466.2 & 1469.2 & 1472.2 & 1475.2 & 1478.2 & 1481.2 & 4.400 \\
\hline 4.500 & 1481.2 & 1484.2 & 1487.2 & 1490.2 & 1493.2 & 1496.2 & 1499.2 & 1502.2 & 1505.2 & 1508.3 & 1511.3 & 4.500 \\
\hline 4.600 & 1511.3 & 1514.3 & 1517.3 & 1520.3 & 1523.3 & 1526.3 & 1529.4 & 1532.4 & 1535.4 & 1538.4 & 1541.5 & 4.600 \\
\hline 4.700 & 1541.5 & 1544.5 & 1547.5 & 1550.5 & 1553.6 & 1556.6 & 1559.6 & 1562.7 & 1565.7 & 1568.7 & 1571.8 & 4.700 \\
\hline 4.800 & 1571.8 & 1574.8 & 1577.9 & 1580.9 & 1583.9 & 1587.0 & 1590.0 & 1593.1 & 1596.1 & 1599.2 & 1602.2 & 4.800 \\
\hline 4.900 & 1602.2 & 1605.3 & 1608.3 & 1611.4 & 1614.4 & 1617.5 & 1620.6 & 1623.6 & 1626.7 & 1629.7 & 1632.8 & 4.900 \\
\hline
\end{tabular}

${ }^{*}$ Based on the International Temperature Scale of 1948. 
TABLE 7-A. Fifty percent iridium-fifty percent rhodium versus iridium thermocouples-Continued

Electromotive force in absolute millivolts. Temperature in degrees $\mathrm{F}^{*}$. Reference junctions at $32{ }^{\circ} \mathrm{F}$.

\begin{tabular}{|c|c|c|c|c|c|c|c|c|c|c|c|c|}
\hline \multirow{2}{*}{$\begin{array}{l}\text { Milli- } \\
\text { volts }\end{array}$} & .000 & .010 & .020 & .030 & .040 & .050 & .060 & .070 & .080 & .090 & .100 & \multirow{2}{*}{$\begin{array}{l}\text { Milli- } \\
\text { volts }\end{array}$} \\
\hline & \multicolumn{11}{|c|}{ Degrees $\mathrm{F}$} & \\
\hline .000 & 1632.8 & 1635.9 & 1638.9 & 1642.0 & 1645. & 1648.2 & 1651.2 & 1654.3 & 1657.4 & 1660.5 & 1663.5 & 5.000 \\
\hline 100 & 1663.5 & 1666.6 & 1669.7 & 1672.8 & .9 & & & & & & & 5.100 \\
\hline 200 & 1694.4 & 1697.5 & 1700.6 & & 170 & 170 & & & & & & 200 \\
\hline 300 & 725.4 & 1728.5 & & & & 174 & & & & & & \\
\hline 400 & 1756.5 & 1759.7 & 1762.8 & & 1769.0 & 1772.2 & & & & & & \\
\hline 5.500 & 1787.8 & 1791.0 & 1794.1 & & & 1803.5 & & 1809.8 & 1812.9 & & & 5.500 \\
\hline 5.600 & 1819.2 & 1822.4 & 1825.5 & 1828.7 & & 1835.0 & & & & & & 5.600 \\
\hline 700 & 1850.8 & & & & & & & & & & & \\
\hline 800 & 1882.5 & & & & & 189 & & & & & & \\
\hline 900 & & & & & & & & & & & & \\
\hline .000 & & & & & & & & & & & & \\
\hline 100 & 978.4 & & & & & & & & & & & 6.100 \\
\hline 200 & & & & & & & & & & & & .200 \\
\hline 300 & 2.9 & 6.1 & & & & & & & & & & 300 \\
\hline 400 & & & & & & & & & & & & 400 \\
\hline 500 & & & & & & & & & & & & .500 \\
\hline 600 & 2140.5 & 2143.8 & 2147.1 & & & & & & & & & 6.600 \\
\hline & 2173.2 & & & & & & & & & & & 6.700 \\
\hline 00 & & & & & & & & & & & & 800 \\
\hline 900 & 2238.9 & 2.2 & & & & & & & & & & 6.900 \\
\hline 000 & & & & & & & & & & & & \\
\hline 7.100 & & & & & & & & & & & & 7.100 \\
\hline 7.200 & & & & & & & & & & & & \\
\hline 300 & & & & & & & & & & & & \\
\hline 400 & & & & & & & & & & & & \\
\hline 7.500 & & & & & & & & & & & & 7.500 \\
\hline 7.600 & 2470.4 & 2473.7 & & & & & & & & & & 7.600 \\
\hline & & & & & & & & & & & & 7.700 \\
\hline 7.800 & & & & & & & & & & & & \\
\hline 7.900 & 2569.8 & & 25 & 2 & & 25 & & 2592.9 & & & 2602.9 & 7.900 \\
\hline 000 & & & & & & & & & & & & \\
\hline & & & & & & & & & & & & 100 \\
\hline 0 & & & & & & & & & & & & \\
\hline 300 & & & & & & & & & & & & \\
\hline & & & & & & & & & & & & 00 \\
\hline .500 & 2767.3 & & & & & & & & & & & 8.500 \\
\hline 600 & & & & & & & & & & & & 8.600 \\
\hline & & & & & & & & & & & & .700 \\
\hline & & & & & & & & & & & & \\
\hline .900 & 2897.3 & & 2903.8 & 2907.0 & & & 2916.7 & 2919.9 & & & 2929.6 & 8900 \\
\hline 000 & & & & & & & & & & & & \\
\hline 9.100 & 2961.7 & & & & & & & & & & & 9.100 \\
\hline & & & & & & & & & & & & \\
\hline & & & & & & & & & & & & \\
\hline & & & & & & & & & & & & \\
\hline & & & & & & & & & & & & 9.500 \\
\hline 9.600 & 3120.3 & & & & & & & & & & & 9.600 \\
\hline 700 & 3151.6 & 3154.7 & 3157.8 & 3160.9 & & & & & & 3179.6 & 3182.7 & 9.700 \\
\hline 9.800 & 3182.7 & & & & & & & & & & & 9.800 \\
\hline & 3213.8 & 3216.9 & 3220.0 & 3223.0 & 3226.1 & 3229.2 & 3232.3 & 3235.4 & 3238.5 & 3241.6 & 3244.7 & 9.900 \\
\hline
\end{tabular}

*Based on the International Temperature Scale of 1948 
TABLE 7-A. Fifty percent iridium-fifty percent rhodium versus iridium thermocouples-Continued Electromotive force in absolute millivolts. Temperature in degrees $\mathrm{F}^{*}$. Reference junctions at $32^{\circ} \mathrm{F}$.

\begin{tabular}{|c|c|c|c|c|c|c|c|c|c|c|c|c|}
\hline \multirow{2}{*}{$\begin{array}{l}\text { Milli- } \\
\text { volts }\end{array}$} & .000 & .010 & .020 & .030 & .040 & .050 & .060 & .070 & .080 & .090 & .100 & \multirow{2}{*}{$\begin{array}{l}\text { Milli- } \\
\text { volts }\end{array}$} \\
\hline & \multicolumn{11}{|c|}{ Degrees F } & \\
\hline 10.000 & 3244.7 & 3247.7 & 3250.8 & 3253.9 & 3257.0 & 3260.0 & 3263.1 & 3266.2 & 3269.3 & 3272.3 & 3275.4 & 10.000 \\
\hline 10.100 & 3275.4 & 3278.5 & 3281.5 & 3284.6 & 3287.7 & 3290.7 & 3293.8 & 3296.8 & 3299.9 & 3302.9 & 3306.0 & 10.100 \\
\hline 10.200 & 3306.0 & 3309.1 & 3312.1 & 3315.2 & 3318.2 & 3321.3 & 3324.3 & 3327.3 & 3330.4 & 3333.4 & 3336.5 & 10.200 \\
\hline 10.300 & 3336.5 & 3339.5 & 3342.5 & 3345.6 & 3348.6 & 3351.7 & 3354.7 & 3357.7 & 3360.7 & 3363.8 & 3366.8 & 10.300 \\
\hline 10.400 & 3366.8 & 3369.8 & 3372.9 & 3375.9 & 3378.9 & 3381.9 & 3384.9 & 3388.0 & 3391.0 & 3394.0 & 3397.0 & 10.400 \\
\hline 10.500 & 3397.0 & 3400.0 & 3403.0 & 3406.0 & 3409.0 & 3412.1 & 3415.1 & 3418.1 & 3421.1 & 3424.1 & 3427.1 & 10.500 \\
\hline 10.600 & 3427.1 & 3430.1 & 3433.1 & 3436.1 & 3439.1 & 3442.1 & 3445.1 & 3448.0 & 3451.0 & 3454.0 & 3457.0 & 10.600 \\
\hline 10.700 & 3457.0 & 3460.0 & 3463.0 & 3466.0 & 3469.0 & 3471.9 & 3474.9 & 3477.9 & 3480.9 & 3483.9 & 3486.8 & 10.700 \\
\hline 10.800 & 3486.8 & 3489.8 & 3492.8 & 3495.8 & 3498.7 & 3501.7 & 3504.7 & 3507.7 & 3510.6 & 3513.6 & 3516.6 & 10.800 \\
\hline 10.900 & 3516.6 & 3519.5 & 3522.5 & 3525.4 & 3528.4 & 3531.4 & 3534.3 & 3537.3 & 3540.2 & 3543.2 & 3546.1 & 10.900 \\
\hline 11.000 & 3546.1 & 3549.1 & 3552.1 & 3555.0 & 3558.0 & 3560.9 & 3563.9 & 3566.8 & 3569.8 & 3572.7 & 3575.6 & 11.000 \\
\hline 11.100 & 3575.6 & 3578.6 & 3581.5 & 3584.5 & 3587.4 & 3590.3 & 3593.3 & 3596.2 & 3599.2 & 3602.1 & 3605.0 & 11.100 \\
\hline 11.200 & 3605.0 & 3608.0 & 3610.9 & 3613.8 & 3616.8 & 3619.7 & 3622.6 & 3625.5 & 3628.5 & 3631.4 & 3634.3 & 11.200 \\
\hline 11.300 & 3634.3 & 3637.2 & 3640.2 & 3643.1 & 3646.0 & 3648.9 & 3651.9 & 3654.8 & 3657.7 & 3660.6 & 3663.5 & 11.300 \\
\hline 11.400 & 3663.5 & 3666.4 & 3669.4 & 3672.3 & 3675.2 & 3678.1 & 3681.0 & 3683.9 & 3686.8 & 3689.7 & 3692.7 & 11.400 \\
\hline 11.500 & 3692.7 & 3695.6 & 3698.5 & 3701.4 & 3704.3 & 3707.2 & 3710.1 & 3713.0 & 3715.9 & 3718.8 & 3721.7 & 11.500 \\
\hline 11.600 & 3721.7 & 3724.6 & 3727.5 & 3730.4 & 3733.3 & 3736.2 & 3739.1 & 3742.0 & 3744.9 & 3747.8 & 3750.7 & 11.600 \\
\hline 11.700 & 3750.7 & 3753.6 & 3756.5 & 3759.4 & 3762.3 & 3765.2 & 3768.1 & 3771.0 & 3773.8 & 3776.7 & 3779.6 & 11.700 \\
\hline 11.800 & 3779.6 & 3782.5 & 3785.4 & 3788.3 & 3791.2 & 3794.1 & 3797.0 & 3799.8 & 3802.7 & 3805.6 & 3808.5 & 11.800 \\
\hline 11.900 & 3808.5 & 3811.4 & 3814.3 & 3817.2 & 3820.0 & 3822.9 & 3825.8 & 3828.7 & 3831.6 & 3834.5 & 3837.4 & 11.900 \\
\hline 12.000 & 3837.4 & 3840.2 & 3843.1 & 3846.0 & 3848.9 & 3851.8 & 3854.6 & 3857.5 & 3860.4 & 3863.3 & 3866.2 & 12.000 \\
\hline 12.100 & 3866.2 & 3869.1 & 3871.9 & 3874.8 & 3877.7 & 3880.6 & 3883.5 & 3886.3 & 3889.2 & 3892.1 & 3895.0 & 12.100 \\
\hline 12.200 & 3895.0 & 3897.8 & 3900.7 & & & & & & & & & 12.200 \\
\hline
\end{tabular}

*Based on the International Temperature Scale of 1948. 
TABLE 8-A. Fifty percent iridium-fifty percent rhodium versus iridium thermocouples

Electromotive force in absolute millivolts. Temperature in degrees $\mathrm{F}^{*}$. Reference junctions at $32{ }^{\circ} \mathrm{F}$.

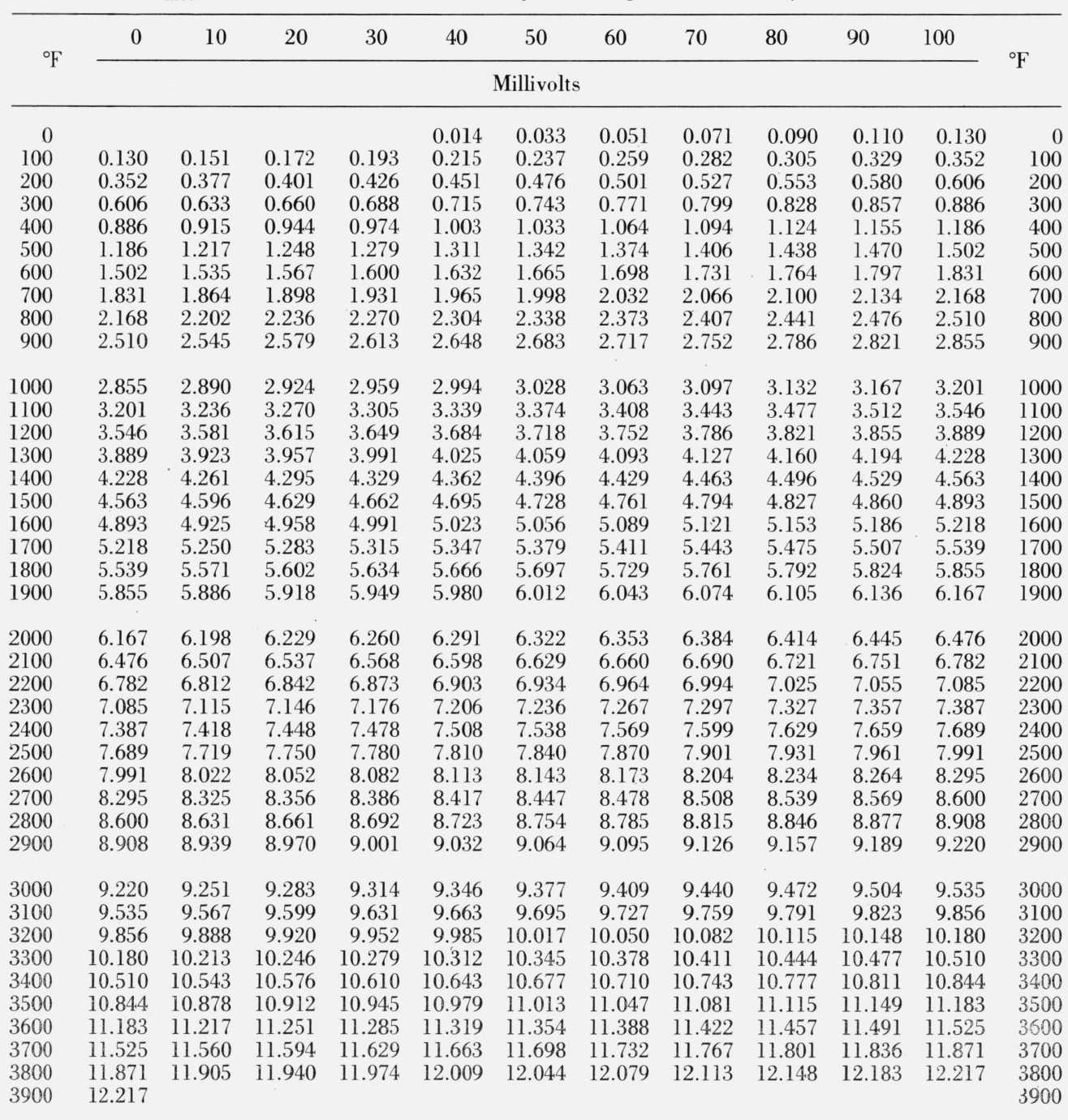

*Based on the International Temperature Scale of 1948. 
TABLE 9-A. Iridium-rhodium versus iridium thermocouples Electromotive force in absolute millivolts

Temperatures in degrees $\mathrm{F}$. Reference junctions at $32^{\circ} \mathrm{F}$.

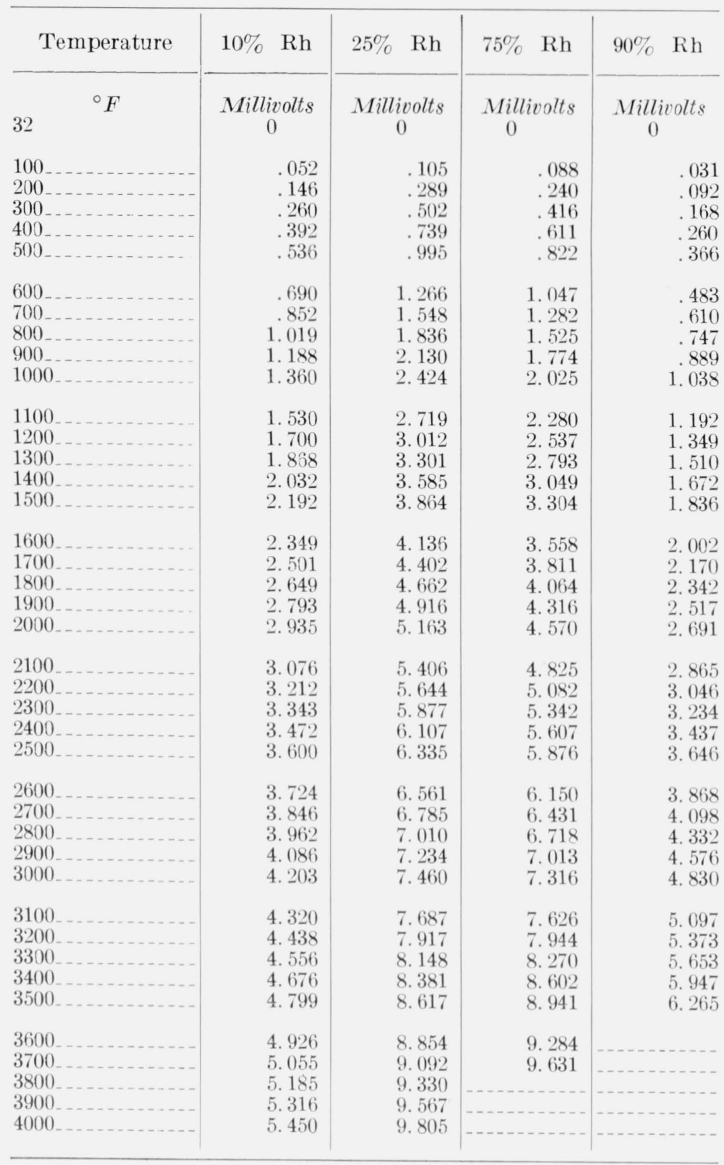

TABLE 10-A. Emf of 50 percent iridium-50 percent rhodium and 60 percent iridium-40 percent rhodium alloys and iridium relative to copper*

\begin{tabular}{|c|c|c|c|}
\hline Temperature & $\begin{array}{c}50 \% \text { Ir }-50 \% \\
\text { Rh vs Cu }\end{array}$ & $\begin{array}{c}60 \% \text { Ir }-40 \% \\
\text { Rh vs Cu }\end{array}$ & $\mathrm{Cu}$ vs Ir \\
\hline $\begin{array}{l}{ }^{\circ} F \\
32 \\
50 \\
75 \\
100 \\
\end{array}$ & $\begin{array}{r}\text { Millivalts } \\
0.000 \\
.027 \\
.067 \\
.107\end{array}$ & $\begin{array}{r}\text { Millivolts } \\
0.000 \\
.026 \\
.064 \\
.103\end{array}$ & $\begin{array}{r}\text { Millivolts } \\
0.000 \\
.006 \\
.014 \\
.023\end{array}$ \\
\hline $\begin{array}{l}125 \\
150 \\
175 \\
200\end{array}$ & $\begin{array}{l}.147 \\
.188 \\
.228 \\
.268\end{array}$ & $\begin{array}{l}.142 \\
.181 \\
.220 \\
.258\end{array}$ & $\begin{array}{l}.035 \\
.049 \\
.065 \\
.084\end{array}$ \\
\hline $\begin{array}{l}225 \ldots \ldots \ldots \\
250 \\
275 \\
300\end{array}$ & $\begin{array}{l}.308 \\
.347 \\
.385 \\
.422\end{array}$ & $\begin{array}{l}.296 \\
.334 \\
.371 \\
.406\end{array}$ & $\begin{array}{l}.105 \\
.129 \\
.155 \\
.184\end{array}$ \\
\hline $\begin{array}{l}350 \ldots \\
400 \ldots\end{array}$ & $\begin{array}{l}.496 \\
.571\end{array}$ & $\begin{array}{l}.477 \\
.549\end{array}$ & $\begin{array}{l}.247 \\
.315\end{array}$ \\
\hline $\begin{array}{l}450 \\
500\end{array}$ & $\begin{array}{l}.638 \\
.701\end{array}$ & $\begin{array}{l}.613 \\
.673\end{array}$ & $\begin{array}{l}.395 \\
.485\end{array}$ \\
\hline
\end{tabular}

${ }^{*}$ These alloys are thermoelectrically positive to copper. Copper is positive to ridium.

\section{References}

[1] G. F. Blackburn and F. R. Caldwell, Reference tables for 40 percent iridium-60 percent rhodium versus iridium thermocouples, J. Res. NBS 66C (Engr. and Instr.) No. 1, 1-12 (Jan.-Mar. 1962); Temperature-Its Measurement and Control in Science and Industry 3 , Part 2 (Reinhold Pub. Corp., New York, N.Y., 1962).

[2] J. Hilsenrath, G. G. Ziegler, C. G. Messina, and P. J Walsh, OMNITAB, a general purpose machine program for the calculation of tables of functions and statistical and numerical analysis of tabular data, to be issued as an NBS publication.

[3] F. R. Caldwell, Thermoelectric properties of platinumrhodium alloys, BS J. Research 10, 373 (1933) RP537.

[4] E. D. Zysk, Private communication, January 20, 1960

[5] J. D. Sine, Pyrometer Supplies Division, MinneapolisHoneywell Regulator Company, Philadelphia, Pa., private communication to F. R. Caldwell (July 1960).

[6] F. R. Caldwell, Thermocouple materials, NBS Mono. 40, March 1, 1962; Temperature-Its Measurement and Control in Science and Industry 3, part 2 (Reinhold Pub. Corp., New York, N.Y., 1962).

[7] W. F. Roeser and S. T. Lonberger, Methods of testing thermocouples and thermocouple materials, NBS Cir. 590 (1958) supersedes R P768).

(Paper 68C1-151) 